\title{
Comparing chemical reaction networks
}

\section{A categorical and algorithmic perspective}

\section{Cardelli, Luca; Tribastone, Mirco; Tschaikowski, Max; Vandin, Andrea}

\section{Published in:}

Theoretical Computer Science

Link to article, DOI:

10.1016/j.tcs.2017.12.018

Publication date:

2019

Document Version

Peer reviewed version

Link back to DTU Orbit

Citation (APA):

Cardelli, L., Tribastone, M., Tschaikowski, M., \& Vandin, A. (2019). Comparing chemical reaction networks: A categorical and algorithmic perspective. Theoretical Computer Science, 765, 47-66.

https://doi.org/10.1016/j.tcs.2017.12.018

\section{General rights}

Copyright and moral rights for the publications made accessible in the public portal are retained by the authors and/or other copyright owners and it is a condition of accessing publications that users recognise and abide by the legal requirements associated with these rights.

- Users may download and print one copy of any publication from the public portal for the purpose of private study or research.

- You may not further distribute the material or use it for any profit-making activity or commercial gain

- You may freely distribute the URL identifying the publication in the public portal

If you believe that this document breaches copyright please contact us providing details, and we will remove access to the work immediately and investigate your claim 


\title{
Comparing chemical reaction networks: A categorical and algorithmic perspective
}

\author{
Luca Cardelli $^{\mathrm{a}}$, Mirco Tribastone ${ }^{\mathrm{b}, *}$, Max Tschaikowski $^{\mathrm{b}}$, Andrea Vandin ${ }^{\mathrm{c}}$ \\ a Microsoft Research \& University of Oxford, UK \\ b IMT School for Advanced Studies Lucca, Italy \\ c DTU Compute, Technical University of Denmark, Lyngby, Denmark
}

\section{A R T I C L E I N F O}

\section{Article history:}

Received 29 March 2017

Received in revised form 16 September

2017

Accepted 27 October 2017

Available online $\mathrm{xxxx}$

\section{Keywords:}

Chemical reaction networks

Bisimulation

Model comparison

Ordinary differential equations

\begin{abstract}
A B S T R A C T
We study chemical reaction networks (CRNs) as a kernel model of concurrency provided with semantics based on ordinary differential equations. We investigate the problem of comparing two CRNs, i.e., to decide whether the solutions of a source and of a target CRN can be matched for an appropriate choice of initial conditions. Using a categorical framework, we extend and unify model-comparison approaches based on dynamical (semantic) and structural (syntactic) properties of CRNs. Then, we provide an algorithm to compare CRNs, running linearly in time with respect to the cardinality of all possible comparisons. Finally, using a prototype implementation, CAGE, we apply our results to biological models from the literature.
\end{abstract}

(C) 2017 Elsevier B.V. All rights reserved.

\section{Introduction}

Chemical reaction networks (CRNs) are a popular concurrency model used in many natural sciences, e.g., chemistry, ecology, epidemiology, and systems biology. Due to the accepted analogy between computational processes and molecular systems [1], more recently they have been receiving increasing attention in computer science. This led, e.g., to foundational results on the expressive power of CRNs (e.g., [2,3]), to languages for the modeling of complex biomolecular systems [4], and model-reduction techniques based on traditional approaches within theoretical computer science such as abstract interpretation [5,6] and bisimulation [7-9].

In this paper we study the problem of comparing the deterministic trajectories of two CRNs. This can be beneficial, e.g., in evolutionary biology, where one is interested in understanding whether a system has evolved into another one preserving the original functionality [10-12]. Another possible application domain is DNA computing, where a specification CRN, which describes the actual dynamics of interest, is compared with an implementation CRN, which takes into account possible physical and technological constraints [13].

We consider the semantics of CRNs based on ordinary differential equations (ODEs), where each species is associated with an ODE describing how its concentration changes over time. By CRN comparison we mean a mapping from the species of a source CRN to those of a target CRN such that related species have same ODE solution at any point in time. This notion, called emulation, has been recently introduced in [10], but no procedure to compute it was provided. Later, it has been shown that emulation can be expressed in terms of backward differential equivalence (BDE) [14], an equivalence relation over

\footnotetext{
* Corresponding author.

E-mail addresses: luca@microsoft.com (L. Cardelli), mirco.tribastone@imtlucca.it (M. Tribastone), max.tschaikowski@imtlucca.it (M. Tschaikowski), anvan@dtu.dk (A. Vandin).
} 
the variables of an ODE system such that related variables have the same ODE solutions whenever initialized equally. This can be seen as a model reduction technique originated in the context of process algebra with ODE semantics [15-17] where suitable behavioral equivalences relate process terms such that the original ODE system can be aggregated into one having one variable per equivalence class [18-22].

Although a partition-refinement algorithm to compute the largest BDE is provided in [14], it cannot be used to find emulations. In fact, not all BDEs represent a mapping from source species into target ones, and hence an emulation: Rather, an emulation is a BDE whose equivalence classes contain exactly one species of the target CRN and at least one of the source CRN. See also [23] for a tutorial-like discussion on the relation among emulation and BDE.

We develop an algorithm to compute all emulations between two CRNs. In addition we study comparisons at the structural/syntactic level, establishing also mappings of reactions from a source CRN to a target one. With reference to the aforementioned evolutionary studies [10,11]. This is useful in practice to trace a certain functionality across two CRNs. From a theoretical point of view, instead, structural relations are of interest because they provide a finitary view for a behavior defined over a continuous state space.

Structure and dynamics have been partially related in [10]. The syntactic notions of stoichiomorphism and reactant morphism provide sufficient conditions to obtain an emulation at the ODE level; however, the converse does not hold. The first contribution of this paper is to develop a new structural notion, called flux morphism, that characterizes emulation. We show this in a categorical setting, proving that the category of CRNs with flux morphisms as arrows is equivalent to that of CRNs where arrows are emulations.

A brute-force approach for finding all emulations between two CRNs could be in principle used by checking all partitions that refine the largest BDE of the union CRN. We avoid such full exploration by using a novel geometric interpretation of BDE, and exploiting the coarsest BDE refinement algorithm [14,9,24] as an inner step. The key idea is that a BDE induces a linear space that can be spanned by an appropriate subset of the generalized eigenvectors belonging to the Jacobian matrix of the ODE system underlying the union CRN. The algorithm begins by building an initial set of so-called guiding partitions from these generalized eigenvectors. (Each such generalized eigenvector induces the finest partition of the species such that two species are in the same block if and only if the corresponding coordinates of the generalized eigenvector are equivalent.)

The intuition is that given a BDE, we can compute a more refined BDE which at the same time refines also a guiding partition. The algorithm, which takes as input any ODE system with totally differentiable drifts, computes all such refinements by recursively visiting the BDE obtained by refining the current one with each guiding partition, starting from the largest BDE of the original ODE system. The properties of the guiding partitions guarantee that the algorithm computes all BDE partitions, among which reside all emulations. In addition, by our characterization result, any emulation found by the algorithm can be always related to structural relations between the two studied CRNs.

Using an implementation of the algorithm integrated in the tool ERODE [25], we apply our framework to a number of CRNs. First we consider networks that result from randomly connecting copies of simpler CRNs. We can explain that these random networks emulate the original one, with the advantage of being less sensitive to perturbations. Then, we consider biological models examined in [10]. We rediscover all the emulations manually derived in [10], and find new ones for models not considered in [10]. Additionally, we exclude the presence of emulations among certain models studied in [10]. For this, we make also a theoretical contribution loosening the dependence of emulation/BDE on the actual choice of the rate parameters. We introduce a class of CRNs, named unimodal influence networks (which include all models of [10] and others in the literature, e.g., [26]), for which the absence of an emulation for a specific choice of rates (i.e., when they are all set to one) implies the absence of emulation for any other choice of rates. Finally, we study the model of a repressilator network [27] from the literature.

Further related work. Several notions of CRN comparison have been recently studied [28-31], but none of them considers rates. Among these, the approach most-closely related to ours is [28]. It establishes sub-graph epimorphisms among graphical representations of CRNs, i.e., mappings from a sub-set of the source species and reactions to all the target ones. A sub-graph epimorphism might imply that the target CRN has been obtained from the source one by merging and deleting nodes and reactions. Instead, emulation accounts only for merging operations. Because of this and the fact that rates and stoichiometries of species in reactions are ignored in the graphical representations, sub-graph epimorphisms do not imply any relation on dynamics. One approach that takes kinetics into account is presented in [13], but this is specific to CRNs implemented using DNA. In addition, the result of correspondence established in [13] is based on an asymptotic fast-slow decomposition of the dynamics whereby fast species are assumed to be found in the stationary regime, while emulation requires equivalent traces at all time points.

BDE generalizes backward bisimulation [9], which offers a polynomial time partition refinement algorithm but is restricted only to elementary CRNs (i.e., with reactions having up to two reagents). We use BDE in this paper because the algorithm for computing all BDEs is defined for the larger class of ODEs with totally differentiable drifts.

Boreale relates bisimulation to linear invariant subspaces for weighted automata [32]. More recently, he proposed in [33] a technique for the minimization of polynomial ODEs in the spirit of BDE which relates linear combinations of trajectories induced by a given input template. Instead, the idea of exploiting geometrical properties of ODEs can be traced back to a seminal work by Li and Rabitz. In [34] they show that aggregations via a linear transformation of the state space can be related to the Jacobian matrix of the ODE system. There are two crucial differences with respect to our approach: (i) The 


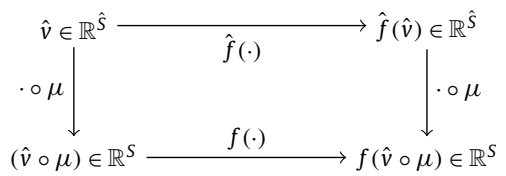

Fig. 1. Graphical representation of the notion of emulation given in Definition 2 (adapted from [10]). The commuting diagram reads from top left (a state $\hat{v}$ of the target drift) towards bottom right (a derivative of the source drift). The diagram states that $(\rightarrow)$ applying the target drift $\hat{f}$ and composing the result with an emulation $\mu$ has the same effect as $(\downarrow)$ composing $\hat{v}$ with $\mu$ and applying the source drift $f$ to the result.

aggregations in [34] concern the derivation of a new ODE system where each variable represents a linear combination of the original ones, loosing information on the dynamics of single variables. Unlike many reductions for CRNs (e.g., [35-37,6]) or control systems (see [38] and references therein) BDE is not an instance of that framework. Hence, its geometric interpretation is a new result in its own right; (ii) The results of [34] are specific to mass-action ODE systems for elementary CRNs, while our algorithm works for totally differentiable drifts. In addition, an implementation of [34] would require symbolic reasoning over infinite sets, which our algorithm can avoid by using the syntactic partition-refinement approach of [9].

The paper extends [39] by publishing all proofs and broadening the application domain with the random CRN examples (Section 5.1) and the repressilator model (Section 5.3). Building on [39], the approach recently presented in [40] using a genetic algorithm in order to compute emulations between unimodal influence networks. This provides a sound but not complete procedure for this class of CRNs, in the sense that the solutions returned are guaranteed to be emulations but the absence of an emulation cannot be proven, unlike with the algorithm herein presented.

Structure of the paper. Section 2 sets the scene by providing the previously established notions of morphism, emulation and BDE [10,14]. Section 3 first introduces the notion of flux morphism and then generalizes all major results of [10,14]. Using those novel results, we then prove that the category of CRNs with flux morphisms as arrows is equivalent to the category of CRNs where arrows are emulations. We continue in Section 4 by providing an algorithm that computes all BDE partitions of an ODE system underlying a totally differentiable drift. This algorithm is then applied in Section 5 to the case studies.

\section{Preliminaries}

Notation. Throughout the paper, $S$ is a finite set of indices. We write $A \rightarrow B$ and $B^{A}$ for the functions from $A$ to $B$. Moreover, we set $\operatorname{dom}(f)=\{a \mid \exists b((a, b) \in f)\}, f(X)=\{f(a) \mid a \in X\}$ and $f^{-1}(Y)=\{a \in \operatorname{dom}(f) \mid f(a) \in Y\}$ for all $X \subseteq$ $\operatorname{dom}(f)$ and $Y \subseteq f(\operatorname{dom}(f))$. A set $X \subseteq \operatorname{dom}(f)$ is called invariant with respect to a function $f$ if $f(X) \subseteq X$.

Comparison of ODE systems. Let the drift $f: \mathbb{R}^{S} \rightarrow \mathbb{R}^{S}$ be totally differentiable. Adopting Newton's notation, $\dot{v}=f(v)$ denotes the ODE system given, in components, by $\dot{v}_{x}=f_{x}(v)$, where $x \in S$. Given an initial condition $v(0) \in \mathbb{R}^{S}$, Picard-Lindelöf's theorem ensures that $\dot{v}=f(v)$ has a unique solution $v: \operatorname{dom}(v) \rightarrow \mathbb{R}^{S}, t \mapsto v(t)$. The assumption $\operatorname{dom}(f)=\mathbb{R}^{S}$ is made to simplify presentation and can be easily removed.

Definition 1 (Backward differential equivalence). Fix a drift $f: \mathbb{R}^{S} \rightarrow \mathbb{R}^{S}$ and a partition $\mathcal{H}$ of $S$. A vector $v \in \mathbb{R}^{S}$ is constant on $\mathcal{H}$ if for all $x, y \in H$ and $H \in \mathcal{H}$ it holds that $v_{x}=v_{y}$. We call $\mathcal{H}$ backward differential equivalent (BDE) if, for any $v \in \mathbb{R}^{S}$ that is constant on $\mathcal{H}$, also $f(v)$ is constant on $\mathcal{H}$.

In $\mathrm{BDE}$, the trajectories of equivalent variables are identical if initialized with the same values.

Theorem 1. A partition $\mathcal{H}$ is BDE if and only if, for any $v(0) \in \mathbb{R}^{S}$ that is constant on $\mathcal{H}$, the solution of $\dot{v}=f(v)$ with initial condition $v(0), v(t)$, is constant on $\mathcal{H}$ for all $t \in \operatorname{dom}(v)$.

Example 1. Let $S=\{x, y\}$, and consider the drift $f_{x}(v)=-2 \cdot v_{x}^{2}+v_{y}^{2}$, and $f_{y}(v)=-2 \cdot v_{y}^{2}+v_{x}^{2}$. Then, the partition $\{\{x, y\}\}$ of $S$ is a BDE, meaning that $v_{x}(t)=v_{y}(t)$ for all $t \geq 0$ whenever $v_{x}(0)=v_{y}(0)$.

Definition 2 (Emulation, adapted from [10]). Fix a source drift $f: \mathbb{R}^{S} \rightarrow \mathbb{R}^{S}$ and target drift $\hat{f}: \mathbb{R}^{\hat{S}} \rightarrow \mathbb{R}^{\hat{S}}$. The surjective function $\mu: S \rightarrow \hat{S}$ is an emulation if $f_{x}(\hat{v} \circ \mu)=\hat{f}_{\mu(x)}(\hat{v})$ for all $\hat{v} \in \mathbb{R}^{\hat{S}}$ and $\hat{x} \in \hat{S}$.

Fig. 1 provides a graphical representation of Definition 2. Note that $(\hat{v} \circ \mu)_{x}=\hat{v}_{\mu(x)}$ for all $x \in S$. Two ODE systems are related by means of an emulation if the trajectories of the source ODE system coincide with those of the target ODE system whenever the initial conditions of both systems are equal with respect to $\mu$, as stated next.

Theorem 2. Let $\mu: S \rightarrow \hat{S}$ be an emulation between the source ODE system $f: \mathbb{R}^{S} \rightarrow \mathbb{R}^{S}$ and the target ODE system $\hat{f}: \mathbb{R}^{\hat{S}} \rightarrow \mathbb{R}^{\hat{S}}$. Then, $v(t)=\hat{v}(t) \circ \mu$ for all $t \geq 0$ whenever $v(0)=\hat{v}(0) \circ \mu$. 
Example 2. Consider $\hat{S}=\{\hat{x}\}$ and $\hat{f}_{\hat{x}}(\hat{v})=-\hat{v}_{\hat{x}}$. Then the function $\mu(x)=\mu(y)=\hat{x}$ is an emulation between $f: \mathbb{R}^{\{x, y\}} \rightarrow$ $\mathbb{R}^{\{x, y\}}$ and $\hat{f}: \mathbb{R}^{\{\hat{x}\}} \rightarrow \mathbb{R}^{\{\hat{x}\}}$, where $f$ is as in Example 1. In particular, it holds that $\hat{v}_{\hat{\chi}}(t)=v_{x}(t)=v_{y}(t)$ for all $t \geq 0$ whenever $\hat{v}_{\hat{x}}(0)=v_{x}(0)=v_{y}(0)$.

The example above indicates that there is a close relation between emulation and BDE, as observed in [9].

Proposition 1. Fix a source drift $f: \mathbb{R}^{S} \rightarrow \mathbb{R}^{S}$, a target drift $\hat{f}: \mathbb{R}^{\hat{S}} \rightarrow \mathbb{R}^{\hat{S}}$ with $S \cap \hat{S}=\emptyset$, and a surjective function $\mu: S \rightarrow \hat{S}$. The partition $\left\{\mu^{-1}(\hat{x}) \cup\{\hat{x}\} \mid \hat{x} \in \hat{S}\right\}$ is a BDE if and only if $\mu$ is an emulation.

Chemical reaction networks. A CRN is a pair $(S, R)$ where $S$ and $R$ are finite sets of species and of reactions, respectively. A reaction is a triple $\rho \rightarrow^{\alpha} \pi$, where $\rho$ and $\pi$ are the multisets of species reactants and products, respectively, and $\alpha>0$ is the reaction rate. We denote by $\rho(x)$ the multiplicity of species $x$ in the multiset $\rho$. The flux stoichiometry $\phi(x, r)$ of a species $x$ in a reaction $r=\left(\rho \rightarrow^{\alpha} \pi\right)$ is defined as $\alpha \cdot(\pi(x)-\rho(x))$. It describes the amount of $x$ transformed through reaction $r$ in a time unit. Any function $\mu: S \rightarrow \hat{S}$ can be lifted to multisets over $S$, e.g., $\mu(x+y)=\mu(x)+\mu(y)$.

The ODE system $\dot{v}=f(v)$ underlying a CRN $(S, R)$ is $f: \mathbb{R}^{S} \rightarrow \mathbb{R}^{S}$, where each component $f_{x}$, with $x \in S$, is:

$$
f_{X}(v):=\sum_{\left(\rho \rightarrow^{\alpha} \pi\right) \in R} \phi\left(x, \rho \rightarrow^{\alpha} \pi\right) \cdot \llbracket \rho \rightarrow^{\alpha} \pi \rrbracket_{v}:=\sum_{\left(\rho \rightarrow \rightarrow^{\alpha} \pi\right) \in R}(\pi(x)-\rho(x)) \cdot \alpha \cdot \prod_{y \in S} v_{y}^{\rho(y)}
$$

Several conventions exist to describe the dynamics of a CRN (see, e.g., [41]). In Equation (1) we adhere to the well-known mass-action kinetics, where the firing rate of a reaction is proportional to the concentrations of the reactants involved. For example, the CRN $\left(\{x, y\},\left\{x+x \rightarrow^{1} y, y+y \rightarrow^{1} x\right\}\right)$ induces the drift of Example 1. Since the ODE system of a CRN is given by polynomials, the drift $f$ is totally differentiable.

Theorem 3 (see [14,9]). For any partition $\mathcal{G}$ of $S$, the coarsest BDE partition $\mathcal{H}$ that refines $\mathcal{G}$ exists and can be calculated using $a$ partition refinement algorithm.

Structural properties of CRNs. Emulation is a dynamical property of an ODE system that is implied by the syntactical properties of CRNs reactant morphism and the stoichiomorphism from [10].

Definition 3. Let $(S, R)$ and $(\hat{S}, \hat{R})$ be the source and the target CRNs, respectively. A pair of functions $(\mu, \sigma) \in(S \rightarrow$ $\hat{S}) \times(R \rightarrow \hat{R})$ is:

- a reactant morphism if, for all $\left(\rho \rightarrow^{\alpha} \pi\right) \in R$ there exist $\hat{\alpha}$ and $\hat{\pi}$ with $\sigma\left(\rho \rightarrow^{\alpha} \pi\right)=\mu(\rho) \rightarrow^{\hat{\alpha}} \hat{\pi} \in \hat{R}$;

- a stoichiomorphism whenever $\sum_{r \in \sigma^{-1}(\hat{r})} \phi(x, r)=\phi(\mu(x), \hat{r})$ for all $x \in S$ and $\hat{r} \in \hat{R}$.

Theorem 4 (see [10]). Fix a source $C R N(S, R)$, a target $C R N(\hat{S}, \hat{R})$ and let $(\mu, \sigma) \in(S \rightarrow \hat{S}) \times(R \rightarrow \hat{R})$ be a reactant morphism and stoichiomorphism. Then, $\mu: S \rightarrow \hat{S}$ is an emulation between the source drift $f$ and the target drift $\hat{f}$.

Category theory. We denote by $|\mathcal{C}|$ the objects of a category $\mathcal{C}$, and by $\operatorname{Hom}_{\mathcal{C}}(a, b)$ the arrows from $a$ to $b$, where $a, b \in|\mathcal{C}|$. A function between categories $\Psi: \mathcal{C} \rightarrow \mathcal{D}$ is a functor if $\Psi(a) \in|\mathcal{D}|$ for all $a \in|\mathcal{C}|$ and $\Psi(\psi): \Psi(a) \rightarrow \Psi(b) \in$ $\operatorname{Hom}_{\mathcal{D}}(\Psi(a), \Psi(b))$ for all $\psi: a \rightarrow b \in \operatorname{Hom}_{\mathcal{C}}(a, b)$. Additionally, $\Psi$ has to preserve identities and compositions.

Definition 4. A functor $\Psi$ establishes the equivalence of categories $\mathcal{C}$ and $\mathcal{D}$ if for any $a, b \in|\mathcal{C}|$ it holds that: (i) the function $\Psi: \operatorname{Hom}_{\mathcal{C}}(a, b) \rightarrow \operatorname{Hom}_{\mathcal{D}}(\Psi(a), \Psi(b))$ is bijective; (ii) and for each $d \in|\mathcal{D}|$ there exists some $c \in|\mathcal{C}|$ such that $d$ is isomorphic to $\Psi(c)$.

\section{Equivalence of structure and dynamics}

Flux morphisms. Ordinary morphisms from [10] are only sufficient conditions for emulation in general.

Example 3. Consider the source CRN $(S, R)=\left(\{x, y, z\},\left\{x+y \rightarrow^{1} x, x+z \rightarrow^{1} x, y+z \rightarrow^{1} y, y+z \rightarrow^{1} 2 z+y\right\}\right)$, and the target $\operatorname{CRN}(\hat{S}, \hat{R})=\left(\{\hat{x}, \hat{y}\},\left\{\hat{x}+\hat{y} \rightarrow^{1} \hat{x}\right\}\right)$. Then, $\mu(x):=\hat{x}$ and $\mu(y):=\mu(z):=\hat{y}$ defines an emulation $\mu: S \rightarrow \hat{S}$. However, there exists no $\sigma: R \rightarrow \hat{R}$ such that $(\mu, \sigma)$ is a reactant morphism. This is because the reactions $y+z \rightarrow^{1} y$ and $y+z \rightarrow{ }^{1} 2 z+y$ are "redundant", i.e., they can be dropped without affecting the underlying drift. At the same time, they introduce the reactant $y+z$ that yields $2 \hat{y}=\mu(y+z) \neq \hat{x}+\hat{y}$.

We tackle this problem by introducing the notion of quotient reactant morphism. 
Definition 5. Two reactions $r_{1}=\left(\rho_{1} \rightarrow^{\alpha_{1}} \pi_{1}\right), r_{2}=\left(\rho_{2} \rightarrow^{\alpha_{2}} \pi_{2}\right) \in R$ of a CRN $(S, R)$ are reactant equivalent, written $r_{1} \sim r_{2}$, if and only if $\rho_{1}=\rho_{2}$. In the following, let $[r]$ denote the equivalence class of $r$ with respect to $\sim$. Moreover, for any given multiset $\rho^{\prime}$, define $R_{\mid \rho^{\prime}}=\left\{\left(\rho \rightarrow^{\alpha} \pi\right) \in R \mid \rho=\rho^{\prime}\right\}$.

Note that $\left[\rho \rightarrow^{\alpha} \pi\right]=R_{\mid \rho}$ for any $\left(\rho \rightarrow^{\alpha} \pi\right) \in R$.

Definition 6. Fix a source CRN $(S, R)$ and a target CRN $(\hat{S}, \hat{R})$. A total surjective function $\mu: S \rightarrow \hat{S}$ and a partial function $\sigma: R / \sim \hookrightarrow \hat{R} / \sim$ form a quotient reactant morphism if

i) for any $\left[\rho \rightarrow^{\alpha} \pi\right] \in R / \sim$, the function $\sigma$ is either undefined if $\hat{R}_{\mid \mu(\rho)}=\emptyset$ or it satisfies

$$
\sigma\left(\left[\rho \rightarrow^{\alpha} \pi\right]\right)=\hat{R}_{\mid \mu(\rho)} \quad \text { when } \quad \hat{R}_{\mid \mu(\rho)} \neq \emptyset
$$

ii) the union of classes for which $\sigma$ is not defined is redundant with respect to $\mu$, i.e., for all $x \in S$ and $\hat{v} \in \mathbb{R}^{\hat{S}}$ it holds that $0=\sum_{r \in R_{0}} \phi(x, r) \cdot \llbracket r \rrbracket_{\hat{v} \circ \mu}$, where $R_{0}=\bigcup\{e \in R / \sim \mid e \notin \operatorname{dom}(\sigma)\}$.

As shown in Example 3, ordinary morphisms from [10] fail in characterizing the notion of emulation because the notion of reactant morphism is unable to identify redundant reactions, i.e. sets of reactions which cumulatively have no effects on the dynamics. As shown in the following example, the notion of quotient reactant morphism solves this problem by relaxing the conditions of reactant morphism.

Example 4. Let $\mu$ be as in Example 3 and $\sigma$ given by $\sigma\left(\left[x+y \rightarrow^{1} x\right]\right)=\sigma\left(\left[x+z \rightarrow^{1} x\right]\right)=\left[\hat{x}+\hat{y} \rightarrow^{1} \hat{x}\right]$, and $\sigma\left(\left[y+z \rightarrow{ }^{1}\right.\right.$ $y])=\emptyset$. Then $(\mu, \sigma)$ is a quotient reactant morphism because $y+z \rightarrow^{1} y$ and $y+z \rightarrow{ }^{1} 2 z+y$ of the source network cancel each other out.

In order to lift Theorem 4 to the new notion of quotient reactant morphism, we have to relax as well the notion of stoichiomorphism.

Definition 7. For a source CRN $(S, R)$ and a target CRN $(\hat{S}, \hat{R})$, the total surjective function $\mu: S \rightarrow \hat{S}$ and the partial function $\sigma: R / \sim \hookrightarrow \hat{R} / \sim$ form a quotient stoichiomorphism if for all $x \in S$ and $\hat{e} \in \hat{R} / \sim$ it holds that

$$
\sum_{e \in \sigma^{-1}(\hat{e})} \sum_{r \in e} \phi(x, r)=\sum_{\hat{r} \in \hat{e}} \phi(\mu(x), \hat{r})
$$

The quotient reactant morphism discussed in Example 4 is easily verified to be a quotient stoichiomorphism.

Definition 8. For a source CRN $(S, R)$ and a target CRN $(\hat{S}, \hat{R})$, a mapping $(\mu, \sigma) \in(S \rightarrow \hat{S}) \times(R / \sim \hookrightarrow \hat{R} / \sim)$ is called flux morphism if $(\mu, \sigma)$ is a quotient reactant morphism and a quotient stoichiomorphism.

The following result shows that a reactant morphism and a stoichiomorphism give rise to a flux morphism.

Theorem 5. If $(\mu, \underline{\sigma}):(S, R) \rightarrow(\hat{S}, \hat{R})$ is a reactant morphism and stoichiomorphism, then $(\mu, \sigma):(S, R / \sim) \rightarrow(\hat{S}, \hat{R} / \sim)$, where $\sigma$ is induced by $\underline{\sigma}$ via $\sigma([r]):=[\underline{\sigma}(r)]$, is a flux morphism.

Proof. Although this result can be shown directly, we give a short proof that uses Theorem 7 presented later. Since ( $\mu, \underline{\sigma})$ is a reactant morphism, we have $\left(\underline{\sigma}\left(\rho \rightarrow^{\alpha} \pi\right)\right)_{\mid 1}=\mu(\rho)$. Hence, $[\underline{\sigma}(r)]=\left[\underline{\sigma}\left(r^{\prime}\right)\right]$ for all $r^{\prime} \in[r]$. Also, $\operatorname{dom}(\sigma)=R / \sim$ because $\operatorname{dom}(\underline{\sigma})=R$. Combining both observations yields $\sigma\left(\left[\rho \rightarrow^{\alpha} \pi\right]\right)=\hat{R}_{\mid \mu(\rho)}$ for all $\left(\rho \rightarrow^{\alpha} \pi\right) \in R$. This shows that $\sigma$ is indeed the unique function characterized by $\mu$ and condition (2). Noting that $(\mu, \underline{\sigma})$ is a reactant morphism and stoichiomorphism, we conclude by using [10] that $\mu$ is an emulation. Theorem 7 ensures then the claim.

The result below, instead, shows that Theorem 4 carries over to flux morphisms. For instance, since $(\mu, \sigma)$ from Example 4 defines a flux morphism, then $\mu: S \rightarrow \hat{S}$ is an emulation.

Theorem 6. Fix a source $C R N(S, R)$, a target $C R N(\hat{S}, \hat{R})$ and let $(\mu, \sigma) \in(S \rightarrow \hat{S}) \times(R / \sim \hookrightarrow \hat{R} / \sim)$ be a flux morphism. Then, $\mu$ is also an emulation, meaning that $f(\hat{v} \circ \mu)=\hat{f}(\hat{v}) \circ \mu$ for all $\hat{v} \in \mathbb{R}^{\hat{S}}$.

Proof. Since $(\mu, \sigma)$ is a quotient stoichiomorphism, we have: $\sum_{e \in \sigma^{-1}(\hat{e})} \sum_{r \in e} \phi(x, r)=\sum_{\hat{r} \in \hat{e}} \phi(\mu(x), \hat{r})$ for all $x \in S$ and $\hat{e} \in \hat{R} / \sim$. This and the fact that $(\mu, \sigma)$ is a quotient reactant morphism imply that 


$$
\sum_{e \in \sigma^{-1}(\hat{e})} \sum_{r \in e} \phi(x, r) \cdot \llbracket r \rrbracket_{\hat{v} \circ \mu}=\sum_{e \in \sigma^{-1}(\hat{e})} \sum_{r \in e} \phi(x, r) \cdot \llbracket \mu\left(r_{\mid 1}\right) \rrbracket_{\hat{v}}=\sum_{\hat{r} \in \hat{e}} \phi(\mu(x), \hat{r}) \cdot \llbracket \hat{r} \rrbracket_{\hat{v}}
$$

for all $\hat{v} \in \mathbb{R}^{\hat{S}}$. Summing over all $\hat{e} \in R / \sim$ yields

$$
\sum_{\hat{e} \in \hat{R} / \sim e \in \sigma^{-1}(\hat{e})} \sum_{r \in e} \phi(x, r) \cdot \llbracket r \rrbracket_{\hat{v} \circ \mu}=\sum_{\hat{e} \in \hat{R} / \sim \hat{r} \in \hat{e}} \sum_{\hat{e}} \phi(\mu(x), \hat{r}) \cdot \llbracket \hat{r} \rrbracket_{\hat{v}}
$$

Thus, if $R_{0}=\bigcup\{e \in R / \sim \mid e \notin \operatorname{dom}(\sigma)\}$ denotes the union of classes for which $\sigma$ is not defined, we observe

$$
\begin{aligned}
\sum_{r \in R} \phi(x, r) \cdot \llbracket r \rrbracket_{\hat{v} \circ \mu} & =0+\sum_{r \in R \backslash R_{0}} \phi(x, r) \cdot \llbracket r \rrbracket_{\hat{v} \circ \mu}=\sum_{\hat{e} \in \hat{R} / \sim e \in \sigma^{-1}(\hat{e})} \sum_{r \in e} \phi(x, r) \cdot \llbracket r \rrbracket_{\hat{v} \circ \mu} \\
& =\sum_{\hat{e} \in \hat{R} / \sim} \sum_{\hat{r} \in \hat{e}} \phi(\mu(x), \hat{r}) \cdot \llbracket \hat{r} \rrbracket_{\hat{v}}=\sum_{\hat{r} \in \hat{R}} \phi(\mu(x), \hat{r}) \cdot \llbracket \hat{r} \rrbracket_{\hat{v}},
\end{aligned}
$$

where the first identity holds because $(\mu, \sigma)$ is a quotient reactant morphism, while the third identity follows from the previous discussion.

The following is a partial converse of Theorem 6: emulation and quotient reactant morphism yield a quotient stoichiomorphism. It is an important step towards our first main result.

Proposition 2. Fix a source $C R N(S, R)$, a target $C R N(\hat{S}, \hat{R})$ and let $(\mu, \sigma) \in(S \rightarrow \hat{S}) \times(R / \sim \hookrightarrow \hat{R} / \sim)$ be a quotient reactant morphism and $\mu$ an emulation. Then, $(\mu, \sigma)$ is also a quotient stoichiomorphism.

Proof. If $R_{0}=\bigcup\{e \in R / \sim \mid e \notin \operatorname{dom}(\sigma)\}$ denotes the union of classes for which $\sigma$ is not defined, we observe that

$$
\sum_{r \in R} \phi(x, r) \cdot \llbracket r \rrbracket_{\hat{v} \circ \mu}=0+\sum_{r \in R \backslash R_{0}} \phi(x, r) \cdot \llbracket r \rrbracket_{\hat{v} \circ \mu}=\sum_{\hat{e} \in \hat{R} / \sim e \in \sigma^{-1}(\hat{e})} \sum_{r \in e} \phi(x, r) \cdot \llbracket r \rrbracket_{\hat{v} \circ \mu}
$$

where the first identity holds true because $(\mu, \sigma)$ is a quotient reactant morphism. Since it is also an emulation, it holds $\sum_{r \in R} \phi(x, r) \cdot \llbracket r \rrbracket_{\hat{v} \circ \mu}=\sum_{\hat{r} \in \hat{R}} \phi(\mu(x), \hat{r}) \cdot \llbracket \hat{r} \rrbracket_{\hat{v}}$ for all $x \in S$ and $\hat{v} \in \mathbb{R}^{\hat{S}}$, thus yielding

$$
\sum_{\hat{e} \in \hat{R} / \sim e \in \sigma^{-1}(\hat{e})} \sum_{r \in e} \phi(x, r) \cdot \llbracket r \rrbracket \hat{v}_{\circ} \mu=\sum_{\hat{r} \in \hat{R}} \phi(\mu(x), \hat{r}) \cdot \llbracket \hat{r} \rrbracket_{\hat{v}}
$$

Now, take any $\hat{e}=\left[\hat{\rho} \rightarrow^{\hat{\alpha}} \hat{\pi}\right] \in \hat{R} / \sim$ and consider the $\hat{v} \in \mathbb{R}^{\hat{S}}$ such that $\hat{v}_{\hat{x}}=0$ if $\hat{\rho}(\hat{x})=0$ and $\hat{v}_{\hat{x}}=1$ otherwise. Then, for any $\hat{r} \in \hat{R}, \llbracket \hat{r} \rrbracket_{\hat{v}}=1$ if $\hat{r}_{\mid 1}=\hat{\rho}$ and $\llbracket \hat{r} \rrbracket_{\hat{v}}=0$ otherwise. Since for the so constructed $\hat{v}$ only the reactions with $\hat{\rho}$ as reagents are left as non-zero summands, we conclude that

$$
\sum_{\hat{r} \in[\hat{\rho} \rightarrow \hat{\alpha} \hat{\pi}]} \sum_{r \in \sigma^{-1}(\hat{r})} \phi(x, r)=\sum_{\hat{r} \in[\hat{\rho} \rightarrow \hat{\alpha} \hat{\pi}]} \phi(\mu(x), \hat{r})
$$

Noting that this holds for all $\hat{e}=\left[\hat{\rho} \rightarrow^{\hat{\alpha}} \hat{\pi}\right] \in \hat{R} / \sim$ and $x \in S$ yields the claim.

We are in a position to state our first main result. It is a converse of Theorem 6. As it has been observed in Example 3, the theorem cannot be stated on the domain of ordinary notions from [10].

Theorem 7. Fix a source $C R N(S, R)$, a target $C R N(\hat{S}, \hat{R})$, and a function $\mu: S \rightarrow \hat{S}$. Then, $\mu$ is an emulation if and only if $(\mu, \sigma)$ is a flux morphism where $\sigma$ is the unique partial function $\sigma: R / \sim \hookrightarrow \hat{R} / \sim$ that satisfies condition (2).

Proof. Since the only if direction holds by Theorem 6, we assume that $\mu: S \rightarrow \hat{S}$ is an emulation and set $\sigma\left(\left[\rho \rightarrow^{\alpha} \pi\right]\right):=$ $\hat{R}_{\mid \mu(\rho)}$ if $\hat{R}_{\mid \mu(\rho)} \neq \emptyset$. Thanks to Proposition 2, it suffices to show that $(\mu, \sigma)$ is a quotient reactant morphism.

Let $f^{\prime}$ denote the aggregated drift underlying $\mu$ that arises from the drift $f$ of $(S, R)$, that is

$$
f_{\mu(x)}^{\prime}(\hat{v})=\sum_{\rho \rightarrow{ }^{\alpha} \pi \in R} \phi(x, r) \cdot \llbracket \mu(\rho) \rrbracket_{\hat{v}} \quad \text { for all } x \in S \text { and } \hat{v} \in \mathbb{R}^{\hat{S}}
$$

Note that $f^{\prime}$ is well-defined because $\mu$ is an emulation, i.e., we have $f_{\mu(x)}^{\prime}(\hat{v})=f_{\mu\left(x^{\prime}\right)}^{\prime}(\hat{v})$ if $\mu(x)=\mu\left(x^{\prime}\right)$. Instead, let $\hat{f}$ denote the drift of $(\hat{S}, \hat{R})$. Since $\mu$ is an emulation, we have $\hat{f}_{\mu(x)}(\hat{v})=f_{x}(\hat{v} \circ \mu)=f_{\mu(x)}^{\prime}(\hat{v})$ for all $x \in S$ and $\hat{v} \in \mathbb{R}^{\hat{S}}$. Hence, 
since two polynomials that coincide on all values need to have the same monomials, we infer that $\hat{f}_{\hat{x}}$ and $f_{\hat{\chi}}^{\prime}$ have the same monomials for all $\hat{x} \in \hat{S}$. By construction, the monomials of $f^{\prime}$ are contained in $\left\{c \cdot \mu(\rho) \mid\left(\rho \rightarrow^{\alpha} \pi\right) \in R, c \in \mathbb{R}\right\}$. Hence, if $\left(\rho \rightarrow^{\alpha} \pi\right) \in R$ and there are no $\hat{\alpha}, \hat{\pi}$ such that $\left(\mu(\rho) \rightarrow^{\hat{\alpha}} \hat{\pi}\right) \in \hat{R}$, we infer that reactions $\left\{\left(\rho^{\prime} \rightarrow^{\alpha^{\prime}} \pi^{\prime}\right) \in R \mid \mu\left(\rho^{\prime}\right)=\mu(\rho)\right\}$ are redundant for all $\hat{v} \circ \mu$ with $\hat{v} \in \mathbb{R}^{\hat{S}}$. This shows that $(\mu, \sigma)$ is a quotient reactant morphism.

Example 5. Let $(\mu, \sigma)$ be as in Example 4. Then, Theorem 7 implies that $\mu: S \rightarrow \hat{S}$ is an emulation from the source to the target network. Conversely, by Theorem 7, it suffices to show that $\mu: S \rightarrow \hat{S}$ is an emulation to infer that the unique $\sigma$ that is induced by condition (2) and $\mu$ is such that $(\mu, \sigma)$ is a flux morphism.

Using Theorem 7 we next prove that flux morphism implies the notion of BDE, and vice versa.

Theorem 8. Fix a source $C R N(S, R)$ and a target $C R N(\hat{S}, \hat{R})$ with $S \cap \hat{S}=\emptyset$. Then, there is a flux morphism $(\mu, \sigma):(S \rightarrow \hat{S}) \times$ $(R / \sim \hookrightarrow \hat{R} / \sim)$ if and only if there exists a $B D E$ partition $\mathcal{H}$ of $S \cup \hat{S}$ such that $|H \cap S| \geq 1$ and $|H \cap \hat{S}|=1$ for all $H \in \mathcal{H}$.

Proof. Let $\mathcal{H}:=\left\{\mu^{-1}(x) \cup\{x\} \mid x \in \hat{S}\right\}$. It is easy to see that there exists an emulation $\mu: S \rightarrow \hat{S}$ iff there exists a BDE partition $\mathcal{H}$ of $S \cup \hat{S}$ such that $|H \cap S| \geq 1$ and $|H \cap \hat{S}|=1$ for all $H \in \mathcal{H}$. Thus, Theorem 7 yields the claim.

Example 6. We have seen that $(\mu, \sigma)$ from Example 4 is a flux morphism and used Theorem 7 in Example 5 to conclude that $\mu: S \rightarrow \hat{S}$ is an emulation. Proposition 1 ensures that $\left\{\mu^{-1}(\hat{x}) \cup\{\hat{x}\} \mid \hat{x} \in \hat{S}\right\}=\{\{x, \hat{x}\},\{y, z, \hat{y}\}\}$ is a BDE partition of the union CRN $(S \cup \hat{S}, R \cup \hat{R})$, thus confirming the if direction of Theorem 8.

We end the paragraph by partially lifting the "change of rates" theorem of [10] to flux morphisms. It states that morphisms respect, to a certain degree, a change of rate coefficients. We first provide two required notions.

Definition 9. Fix a CRN $(S, R)$. A bijection $\iota: R \rightarrow R^{\prime}$ that satisfies $\iota\left(\rho \rightarrow^{\alpha} \pi\right)=\rho \rightarrow^{\alpha^{\prime}} \pi$ for all $\left(\rho \rightarrow^{\alpha} \pi\right) \in R$ is called a change of rates.

Definition 10. We call a set of reactions $\sim$-uniform if any two reactions $\left(\rho_{1} \rightarrow^{\alpha_{1}} \pi_{1}\right),\left(\rho_{2} \rightarrow^{\alpha_{2}} \pi_{2}\right) \in R$ satisfy $\alpha_{1}=\alpha_{2}$ if $\rho_{1}=\rho_{2}$. A change of rates is called $\sim$-uniform if it leads to a $\sim$-uniform set of reactions.

Theorem 9. Fix a source $C R N(S, R)$, a target $C R N(\hat{S}, \hat{R})$ with $\hat{R}$ being $\sim$-uniform, and let $(\mu, \sigma) \in(S \rightarrow \hat{S}) \times(R / \sim \hookrightarrow \hat{R} / \sim)$ be a flux morphism. Then, for any $\sim$-uniform change of rates $\hat{\imath}: \hat{R} \rightarrow \hat{R}^{\prime}$, there exists a change of rates $\iota: R \rightarrow R^{\prime}$ such that $\left(\mu, \sigma^{\prime}\right)$ : $\left(S, R^{\prime}\right) \hookrightarrow\left(\hat{S}, \hat{R}^{\prime}\right)$, where $\sigma^{\prime}$ is induced by $\mu: S \rightarrow \hat{S}$ and condition (2), is a flux morphism.

Proof. For any $\left(\rho \rightarrow^{\alpha} \pi\right) \in R \backslash \operatorname{dom}(\sigma)$, we set $\iota^{\prime}\left(\rho \rightarrow^{\alpha} \pi\right):=\rho \rightarrow^{\alpha} \pi$. Since a change of rates affects only rates, this implies that $R^{\prime} \backslash \operatorname{dom}\left(\sigma^{\prime}\right)=R \backslash \operatorname{dom}(\sigma)$ is redundant with respect to $\hat{v} \circ \mu$ for all $\hat{v} \in \mathbb{R}^{\hat{S}}$, thus showing that $\left(\mu, \sigma^{\prime}\right)$ is a quotient reactant morphism. We are left with choosing the remaining rate assignments of $\iota^{\prime}$ such that $\left(\mu, \sigma^{\prime}\right):\left(S, R^{\prime}\right) \hookrightarrow\left(\hat{S}, \hat{R}^{\prime}\right)$ is a quotient stoichiomorphism. Since $(\mu, \sigma)$ is a quotient stoichiomorphism, we know that

$$
\sum_{e \in \sigma^{-1}(\hat{e})} \sum_{r \in e} \underbrace{\left(\pi_{r}(x)-\rho_{r}(x)\right) \cdot \alpha_{r}}_{=\phi(x, r)}=\sum_{\hat{r} \in \hat{e}} \underbrace{\left(\hat{\pi}_{\hat{r}}(\mu(x))-\hat{\rho}_{\hat{r}}(\mu(x))\right) \cdot \hat{\alpha}_{\hat{r}}}_{=\phi(\mu(x), \hat{r})}
$$

By assumption, this simplifies to

$$
\sum_{e \in \sigma^{-1}(\hat{e})} \sum_{r \in e}\left(\pi_{r}(x)-\rho_{r}(x)\right) \cdot \alpha_{r}=\sum_{\hat{r} \in \hat{e}}\left(\hat{\pi}_{\hat{r}}(\mu(x))-\hat{\rho}_{\hat{r}}(\mu(x))\right) \cdot \hat{\alpha}_{\hat{e}}
$$

Recall that we have assumed that a reaction can appear at most once in a set of reactions. Hence, by indexing quantities by $r$ (and $\hat{r}$ ), we can keep track of the stoichiometries and reaction coefficients. By applying $\hat{\imath}$ to $\hat{R}$, the quantity $\sum_{\hat{r} \in \hat{e}}\left(\hat{\pi}_{\hat{r}}(\mu(x))-\right.$ $\left.\hat{\rho}_{\hat{r}}(\mu(x))\right) \cdot \hat{\alpha}_{\hat{e}}$ is changed to $\sum_{\hat{r} \in \hat{e}}\left(\hat{\pi}_{\hat{r}}(\mu(x))-\hat{\rho}_{\hat{r}}(\mu(x))\right) \cdot \hat{\alpha}_{\hat{e}}^{\prime}$. It suffices to show that, for all $\hat{e} \in \hat{R} / \sim$, the coefficients $\left\{\alpha_{r} \mid r \in\right.$ $\left.\bigcup \sigma^{-1}(\hat{e})\right\}$ can be replaced by the coefficients $\left\{\alpha_{r}^{\prime} \mid r \in \bigcup \sigma^{-1}(\hat{e})\right\}$ such that

$$
\sum_{e \in \sigma^{-1}(\hat{e})} \sum_{r \in e}\left(\pi_{r}(x)-\rho_{r}(x)\right) \cdot \alpha_{r}^{\prime}=\sum_{\hat{r} \in \hat{e}}\left(\hat{\pi}_{\hat{r}}(\mu(x))-\hat{\rho}_{\hat{r}}(\mu(x))\right) \cdot \hat{\alpha}_{\hat{e}}^{\prime}
$$

From above, it is easy to see that setting $\alpha_{r}^{\prime}:=\alpha_{r} \cdot \frac{\hat{\alpha}_{\hat{e}}^{\prime}}{\hat{\alpha}_{\hat{e}}}$ does the job. 
In [10] no assumptions were made on the nature of rate change. This comes, however, at the price of making the stronger assumption of ordinary morphisms. Instead, the $\sim$-uniformity assumption on the rate change cannot be dropped from Theorem 9. Consider, for instance, the source CRN $\left(\{x\},\left\{x \rightarrow{ }^{\alpha_{1}} x, x \rightarrow \rightarrow^{\alpha_{2}} 2 x\right\}\right)$ and the target one $\left(\{\hat{x}\},\left\{\hat{x} \rightarrow{ }^{\beta_{1}} \emptyset, \hat{x} \rightarrow{ }^{\beta_{2}} 3 \hat{x}\right\}\right)$. If $\beta_{1}=\beta_{2}=\beta$, setting $\alpha_{1}=\alpha_{2}=\beta$ induces a quotient stoichiomorphism. Instead, if $\beta_{1}=10$ and $\beta_{2}=1$, there is no pair $\alpha_{1}, \alpha_{2}>0$ for which there exists a quotient stoichiomorphism.

Equivalence of structure and dynamics. We now build on our findings to establish an equivalence result between the category of structurally related networks $\mathcal{C}^{m}$ and the category of dynamically related networks $\mathcal{C}^{e}$.

Definition 11. The category $\mathcal{C}^{m}$ has CRNs as objects and flux morphisms as arrows. The identity morphism of (S,R) is the pair of identity functions $\left(\mathrm{id}_{S}, \mathrm{id}_{R}\right)$, while the composition of two flux morphisms $(\mu, \sigma):(S, R) \rightarrow(\hat{S}, \hat{R})$ and $(\hat{\mu}, \hat{\sigma})$ : $(\hat{S}, \hat{R}) \rightarrow\left(\hat{S}^{\prime}, \hat{R}^{\prime}\right)$ is given by $(\hat{\mu}, \hat{\sigma}) \circ(\mu, \sigma)\left(x,\left[\rho \rightarrow^{\alpha} \pi\right]\right):=\left(\hat{\mu}(\mu(x)), \hat{R}_{\mid \hat{\mu}(\mu(\rho))}^{\prime}\right)$.

The definition of $\mathcal{C}^{e}$ is straightforward and follows next.

Definition 12. The category $\mathcal{C}^{e}$ has CRNs as objects and emulations as arrows. The identity emulation is given by the identity function $\mathrm{id}_{S}$ and the composition of emulations is defined in the obvious way.

The next result ensures that $\mathcal{C}^{m}$ and $\mathcal{C}^{e}$ are indeed categories.

Lemma 1. Emulations and flux morphisms are closed under composition.

Proof. It can be easily seen that a composition of emulations is again an emulation, so let us fix flux morphisms $(S, R) \stackrel{(\mu, \sigma)}{\longrightarrow}(\hat{S}, \hat{R}),(\hat{S}, \hat{R}) \stackrel{(\hat{\mu}, \hat{\sigma})}{\longrightarrow}\left(\hat{S}^{\prime}, \hat{R}^{\prime}\right)$. Theorem 6 implies that $\hat{\mu}$ and $\mu$ are emulations, thus ensuring that $\hat{\mu} \circ \mu$ is an emulation as well. The claim follows then by Theorem 7 .

Category $\mathcal{C}^{m}$ relates CRNs that share the same structure, while $\mathcal{C}^{e}$ regards $C R N s$ related by emulations. We relate now both categories by means of a functor.

Definition 13. Set $\Psi: \mathcal{C}^{m} \rightarrow \mathcal{C}^{e}$ by $\Psi((S, R))=(S, R)$ for all $(S, R) \in\left|\mathcal{C}^{m}\right|$ and by $\Psi((\mu, \sigma))=\mu$ for all $(\mu, \sigma) \in$ $\operatorname{Hom}_{\mathcal{C}^{m}}((S, R),(\hat{S}, \hat{R}))$ and $(S, R),(\hat{S}, \hat{R}) \in\left|\mathcal{C}^{m}\right|$.

Note that $\Psi$ is a well-defined functor because Theorem 6 ensures that $\mu$ is an emulation if $(\mu, \sigma)$ is a flux morphism.

The next result states that by studying structural properties of CRNs one does not lose symmetries at the ODE level. It thus formally shows the equivalence of structure and function on the level of CRNs in [10].

Theorem 10. The functor $\Psi: \mathcal{C}^{m} \rightarrow \mathcal{C}^{e}$ yields the equivalence of $\mathcal{C}^{m}$ and $\mathcal{C}^{e}$.

Proof. Let us fix two $(S, R),(\hat{S}, \hat{R}) \in\left|\mathcal{C}^{m}\right|$. Given that $\Psi$ is well-defined, we are left with proving that

$$
\Psi: \operatorname{Hom}_{\mathcal{C}^{m}}((S, R),(\hat{S}, \hat{R})) \rightarrow \operatorname{Hom}_{\mathcal{C}^{e}}((S, R),(\hat{S}, \hat{R}))
$$

is bijective. To this end, fix some emulation $\mu: S \rightarrow \hat{S} \in \operatorname{Hom}_{\mathcal{C}^{e}}((S, R),(\hat{S}, \hat{R}))$. Note that $\mu: S \rightarrow \hat{S}$ and $(2)$ imply that there can be at most one $\sigma: R / \sim \hookrightarrow \hat{R} / \sim \operatorname{such}$ that $(\mu, \sigma)$ is a flux morphism. Theorem 7 ensures that this candidate function $\sigma$ does the job. Since $\left(\operatorname{id}_{S}, \operatorname{id}_{R}\right):(S, R) \rightarrow(S, R)$ is an isomorphism, $\left|\mathcal{C}^{e}\right|=\left|\mathcal{C}^{m}\right|$ and $\Psi((S, R))=(S, R)$, the second property of Definition 4 is trivially fulfilled and the proof is complete.

\section{Algorithmic model comparison}

In this section we present an algorithm for the calculation of all BDE partitions underlying a totally differentiable drift $f: \mathbb{R}^{S} \rightarrow \mathbb{R}^{S}$. In addition to being of importance on its own in the area of model reduction, it can be used to decide whether a source ODE system $f$ and a target ODE system $\hat{f}$ are related by means of an emulation. In particular, the algorithm provides a technique to decide whether there exists an arrow between two given CRNs in $\mathcal{C}^{e}$ (and, thanks to Theorem 10, also in $\mathcal{C}^{m}$ ). In the case there are arrows, the algorithm calculates all of them.

We reiterate that the partition refinement algorithm of Theorem 3 cannot be used to tackle the problem because it calculates the coarsest BDE partition but does not tell one whether this partition can be split further into finer BDE partitions. In particular, the number of possible refinements of the coarsest BDE partition is still too large to be analyzed efficiently. As discussed in Section 1, the main idea behind our algorithm is to find a set of guiding partitions which, if applied to the partition refinement algorithm, guarantees to find any BDE partition. We thus perform a guided, instead of a brute-force, search. 
Calculating all BDEs. We first introduce the set of vectors $\mathcal{U}_{\mathcal{H}}$ that is constant on a given partition $\mathcal{H}$ of $S$ and observe that $\mathcal{U}_{\mathcal{H}}$ is a linear subspace of $\mathbb{R}^{S}$.

Lemma 2. Fix a partition $\mathcal{H}$ of $S$. Then, the set $\mathcal{U}_{\mathcal{H}}:=\left\{v \in \mathbb{R}^{S} \mid v\right.$ is constant on $\left.\mathcal{H}\right\}$ is a linear subspace of $\mathbb{R}^{S}$.

Proof. We have to check that $\mathcal{U}_{\mathcal{H}}$ satisfies the three axioms of a vector space. Obviously, $0 \in \mathbb{R}^{S}$ is constant on any partition, so $0 \in \mathcal{U}_{\mathcal{H}}$. Also, $\alpha \cdot v$ is constant on $\mathcal{H}$ for any $\alpha \in \mathbb{R}$ when $v$ is constant on $\mathcal{H}$. Similarly, $v+w$ is constant on $\mathcal{H}$ whenever $v, w$ are constant on $\mathcal{H}$.

For convenience, we write space instead of linear space. The first step towards our algorithm is to observe that, whenever $\mathcal{H}$ is a BDE partition, $\mathcal{U}_{\mathcal{H}}$ is an invariant space of the Jacobi matrix of $f$ evaluated at point $\mathbb{1} \in \mathbb{R}^{S}$, written $J(\mathbb{1})$, where $\mathbb{1}_{x}=1$ for all $x \in S$. This observation is inspired by [34], where subspaces underlying linear transformations of ODE state spaces are shown to be invariant sets of the transpose of $J(\mathbb{1})$.

Theorem 11. Fix a totally differentiable $f: \mathbb{R}^{S} \rightarrow \mathbb{R}^{S}$ and assume that $\mathcal{H}$ is a BDE partition of $S$. It holds that $J(\mathbb{1}) v \in \mathcal{U}_{\mathcal{H}}$ for any $v \in \mathcal{U}_{\mathcal{H}}$, meaning that $\mathcal{U}_{\mathcal{H}}$ is an invariant space of the Jacobi matrix of $f$ evaluated at $\mathbb{1} \in \mathbb{R}^{S}$.

Proof. Recall that for a given drift $f: \mathbb{R}^{S} \rightarrow \mathbb{R}^{S}, v \mapsto f(v)$, a partition $\mathcal{H}$ of $S$ is BDE if and only if $f(v)$ is constant on $\mathcal{H}$ whenever $v$ is constant on $\mathcal{H}$. Since $f$ is totally differentiable, there exists a continuous function $r: \mathbb{R}^{S} \rightarrow \mathbb{R}^{S}, v \mapsto r(v)$ such that $f(\mathbb{1}+v)=f(\mathbb{1})+J(\mathbb{1}) \cdot v+r(v)$ for all $v \in \mathbb{R}^{S}, r(0)=0$ and $\lim _{v \rightarrow 0} \frac{r(v)}{\|v\|}=0$. (We use the symbol $\cdot$ to denote matrix multiplication and identify vectors as matrices with one column. Note also that $v \mapsto f(\mathbb{1})+J(\mathbb{1}) \cdot v$ is the linearization of $f$ at point 1.) Fix arbitrary $x, y \in H$ and $H \in \mathcal{H}$. Then, in the case $v \in \mathcal{U}_{\mathcal{H}}$, the above discussion implies that

$$
f_{x}(\mathbb{1}+v)-f_{y}(\mathbb{1}+v)=f_{x}(\mathbb{1})-f_{y}(\mathbb{1})+(J(\mathbb{1}) \cdot v)_{x}-(J(\mathbb{1}) \cdot v)_{y}+r_{x}(v)-r_{y}(v),
$$

thus yielding

$$
\frac{r_{y}(v)-r_{x}(v)}{\|v\|}=\frac{1}{\|v\|}\left((J(\mathbb{1}) \cdot v)_{x}-(J(\mathbb{1}) \cdot v)_{y}\right)=\left(J(\mathbb{1}) \cdot \frac{v}{\|v\|}\right)_{x}-\left(J(\mathbb{1}) \cdot \frac{v}{\|v\|}\right)_{y}
$$

Since $v \in \mathcal{U}_{\mathcal{H}}$ can be chosen arbitrarily small, this implies that $(J(\mathbb{1}) \cdot v)_{x}=(J(\mathbb{1}) \cdot v)_{y}$ for all $v \in \mathcal{U}_{\mathcal{H}}$. Hence, $\mathcal{U}_{\mathcal{H}}$ is an invariant space of $J(\mathbb{1})$ for any $\mathbb{1} \in \mathcal{U}_{\mathcal{H}}$. Since $\mathbb{1} \in \mathcal{U}_{\mathcal{H}}$, this yields the claim.

Example. Set $S=\{1,2,3\}$ and consider the drift $f: \mathbb{R}^{S} \rightarrow \mathbb{R}^{S}$

$$
f_{1}(v)=-v_{1} v_{3}+v_{3} v_{2} \quad f_{2}(v)=-v_{2} v_{1}+v_{1} v_{3} \quad f_{3}(v)=-v_{3} v_{2}+v_{2} v_{1}
$$

The Jacobi matrix at point $\mathbb{1}$ is then

$$
J(\mathbb{1})=\left(\begin{array}{ccc}
-1 & 1 & 0 \\
0 & -1 & 1 \\
1 & 0 & -1
\end{array}\right)
$$

With this, it holds that $J(\mathbb{1}) \cdot(1,1,1)^{T}=(0,0,0)^{T}$, thus showing that the subspace $\left\{\eta \cdot(1,1,1)^{T} \mid \eta \in \mathbb{R}\right\}$ of $\mathbb{R}^{3}$ is an invariant space of $J(\mathbb{1})$.

Having established that any $\mathcal{U}_{\mathcal{H}}$ of a BDE partition $\mathcal{H}$ is an invariant set of $J(\mathbb{1})$, we now discuss how to calculate the invariant subspaces of $J(\mathbb{1})$. This is a classic topic of linear algebra, so let us recall some elementary notions.

Definition 14. Fix $A \in \mathbb{R}^{S \times S}$. If $\lambda \in \mathbb{R}$ and $w \in \mathbb{R}^{S} \backslash\{0\}$ are such that $A w=\lambda w$, we call $\lambda$ the eigenvalue corresponding to the eigenvector $w$. The set of all eigenvalues of $A$ is called spectrum and is denoted by $\sigma(A)$. The eigenspace of $\lambda \in \sigma(A)$ is given by $E_{\lambda}(A)=\left\{w \in \mathbb{R}^{S} \mid(A-\lambda I) w=0\right\}$. The matrix $A$ is diagonalizable if there exists a basis of $\mathbb{R}^{S}$ consisting of eigenvectors of $A$.

In order to simplify presentation, we first discuss the case where $J(\mathbb{1})$ is diagonalizable. Let us assume that $\mathcal{W}$ is an invariant set of $J(\mathbb{1})$. Since $J(\mathbb{1})$ is diagonalizable and $E_{\lambda}(J(\mathbb{1})) \cap E_{\lambda^{\prime}}(J(\mathbb{1}))=\{0\}$ whenever $\lambda \neq \lambda^{\prime}, \mathbb{R}^{S}$ can be decomposed into the eigenspaces of $J(\mathbb{1})$, i.e. $\mathbb{R}^{S}=\bigoplus_{\lambda} E_{\lambda}(J(\mathbb{1}))$. Moreover, since this implies that $\mathcal{W}=\bigoplus_{\lambda}\left(\mathcal{W} \cap E_{\lambda}(J(\mathbb{1}))\right.$ ), we infer that any invariant set of $J(\mathbb{1})$ can be written as a direct sum of subsets of eigenspaces.

At the same time, any subspace of an eigenspace $E_{\lambda}(J(\mathbb{1}))$ is an invariant space of $J(\mathbb{1})$ because $A w=\lambda w$ for all $w \in E_{\lambda}(J(\mathbb{1}))$. Thus, it suffices to determine the set of all possible subspaces of $E_{\lambda}(J(\mathbb{1}))$. To get an idea on how those look like, let us fix some $\lambda \in \sigma(J(\mathbb{1}))$ and assume that $E_{\lambda}(J(\mathbb{1}))=\left\langle u_{1}, u_{2}, u_{3}\right\rangle$, where $\left\langle w_{1}^{\prime}, \ldots, w_{k}^{\prime}\right\rangle$ denotes the set of all 
linear combinations $\sum_{i=1}^{k} \eta_{i} w_{i}^{\prime}$. The one dimensional invariant sets contained in $E_{\lambda}(J(\mathbb{1}))$ are then given by the family $\left\langle\eta_{1} u_{1}+\eta_{2} u_{2}+\eta_{3} u_{3}\right\rangle$ where $\eta_{1}, \eta_{2}, \eta_{3} \in \mathbb{R}$ such that $\left|\eta_{1}\right|+\left|\eta_{2}\right|+\left|\eta_{3}\right| \neq 0$. Instead, the two dimensional invariant sets of $E_{\lambda}(J(\mathbb{1}))$ are given by the families $\left\langle\eta u_{1}+\eta^{\prime} u_{2}, u_{3}\right\rangle,\left\langle\eta u_{1}+\eta^{\prime} u_{3}, u_{2}\right\rangle$ and $\left\langle u_{1}, \eta u_{2}+\eta^{\prime} u_{3}\right\rangle$ with $|\eta|+\left|\eta^{\prime}\right| \neq 0$. Finally, there is only one three dimensional invariant set, namely $E_{\lambda}(J(\mathbb{1}))=\left\langle u_{1}, u_{2}, u_{3}\right\rangle$ itself.

The above discussion leads to the following.

Theorem 12. Let $J(\mathbb{1})$ be diagonalizable. Then, for any $B D E$ space $\mathcal{U}_{\mathcal{H}}$, there are linearly independent vectors $w_{1}^{\lambda}, \ldots, w_{k_{\lambda}}^{\lambda} \in E_{\lambda}(J(\mathbb{1}))$ with $0 \leq k_{\lambda} \leq \operatorname{dim} E_{\lambda}(J(\mathbb{1}))$ such that $\mathcal{U}_{\mathcal{H}}=\bigoplus_{\lambda}\left\langle w_{1}^{\lambda}, \ldots, w_{k_{\lambda}}^{\lambda}\right\rangle$.

Proof. The proof is straightforward, and hence it is omitted.

Thus, if we fix for each $\lambda \in \sigma(J(\mathbb{1}))$ a basis $u_{1}^{\lambda}, \ldots, u_{\operatorname{dim} E_{\lambda}}^{\lambda} \in \mathbb{R}^{S}$ of $E_{\lambda}(J(\mathbb{1}))$, then for any $w_{i}^{\lambda}$ there exist coefficients $\eta_{j}^{(\lambda, i)} \in \mathbb{R}$ so that $w_{i}^{\lambda}=\sum_{j=1}^{\operatorname{dim} E_{\lambda}} \eta_{j}^{(\lambda, i)} u_{j}^{\lambda}$.

We now drop the assumption of $J(\mathbb{1})$ being diagonalizable. To this end, from now on until Theorem 14, we work on $\mathbb{C}^{S}$ as a vector space over the field $\mathbb{C}$.

Definition 14 carries over to the complex case by replacing each $\mathbb{R}$ with $\mathbb{C}$. In particular, $\sigma(A) \subseteq \mathbb{C}$.

Definition 15. For $A \in \mathbb{C}^{S \times S}$, the generalized eigenspace of $\lambda \in \sigma(A)$ is $E_{\lambda}^{*}(A)=\left\{z \in \mathbb{C}^{n} \mid(A-\lambda I)^{\nu_{\lambda}} z=0\right\}$, where $v_{\lambda}$ denotes the algebraic multiplicity of $\lambda$. Call any $z \in E_{\lambda}^{*}(A) \backslash\{0\}$ a generalized eigenvector of $A$.

It can be shown that $E_{\lambda}^{*}(A)$ is an invariant set of $A$ for any $\lambda \in \sigma(A)$ and that $\mathbb{C}^{n}=\bigoplus_{\lambda} E_{\lambda}^{*}(A)$ over field $\mathbb{C}$. Moreover, the following well-known result holds.

Theorem 13. A matrix $A \in \mathbb{C}^{S \times S}$ is in Jordan normal form if all entries not on the diagonal and the superdiagonal are zero and

$$
A=\left(\begin{array}{ccc}
A_{1} & & \\
& \ddots & \\
& & A_{p}
\end{array}\right), A_{i}=\left(\begin{array}{cccc}
\lambda & 1 & & \\
& \lambda & \ddots & \\
& & \ddots & 1 \\
& & & \lambda
\end{array}\right)
$$

where $\lambda \in \sigma(A)$. It can be shown that for any $A \in \mathbb{C}^{S \times S}$ there exists a basis $B \in \mathbb{C}^{S \times S}$ of $\mathbb{C}^{S}$ (over field $\mathbb{C}$ ) consisting of generalized eigenvectors of $A$ such that $B^{-1} A B$ is in Jordan normal form.

Proof. The result can be found in most monographs about linear algebra, e.g., [42, Chapter 6].

Let $\Re(z)$ and $\Im(z)$ denote the real and imaginary part of any $z \in \mathbb{C}$, respectively, and $\mathbb{H}=\{(x, y) \in \mathbb{C} \mid y \geq 0\}$ be the upper half plane of $\mathbb{C}$. We continue by generalizing Theorem 12 .

Theorem 14. Let $A \in \mathbb{R}^{S \times S}$ and let $B^{-1} A B$ be a Jordan normal form of $A$. Let $B_{\lambda}=\left\{z_{1}^{\lambda}, \ldots, z_{\mathrm{dim}}^{\lambda} E_{\lambda}^{*} \subseteq \mathbb{C}^{S}\right.$ be the generalized eigenvectors from $B$ that span $E_{\lambda}^{*}(A)$ over field $\mathbb{C}$. With

$$
B_{\lambda}^{\prime}:=\left\{\Re\left(z_{i}^{\lambda}\right) \mid z_{i}^{\lambda} \in B_{\lambda}\right\} \cup\left\{\Im\left(z_{i}^{\lambda}\right) \mid z_{i}^{\lambda} \in B_{\lambda}\right\}
$$

for all $\lambda \in \sigma(J(\mathbb{1})) \cap \mathbb{H}$, let $\mathcal{B}_{\lambda}=\left\{u_{1}^{\lambda}, \ldots, u_{d_{\lambda}}^{\lambda}\right\}$ be a basis of $\left\langle B_{\lambda}^{\prime}\right\rangle$ over field $\mathbb{R}$. Then, for any BDE space $\mathcal{U}_{\mathcal{H}}$, there are linearly independent vectors $w_{1}^{\lambda}, \ldots, w_{k_{\lambda}}^{\lambda} \in\left\langle\mathcal{B}_{\lambda}\right\rangle$ over field $\mathbb{R}$ with $0 \leq k_{\lambda} \leq d_{\lambda}$ so that $\mathcal{U}_{\mathcal{H}}=\bigoplus_{\lambda}\left\langle w_{1}^{\lambda}, \ldots, w_{k_{\lambda}}^{\lambda}\right\rangle$ over field $\mathbb{R}$.

Proof. The result relies on a special version of Jordan decomposition that is used in the field of dynamical systems, see Section 1.8 in [43]. It implies that $\mathcal{B}:=\bigcup_{\lambda} \mathcal{B}_{\lambda}$ is a basis of $\mathbb{R}^{S}$ and that $J(\mathbb{1})$ attains an almost diagonal form if we work on the basis $\mathcal{B}$ instead of the canonical one. This nice form implies then that $\mathcal{U}_{\mathcal{H}}=\bigoplus_{\lambda}\left\langle w_{1}^{\lambda}, \ldots, w_{k_{\lambda}}^{\lambda}\right\rangle$ with $w_{1}^{\lambda}, \ldots, w_{k_{\lambda}}^{\lambda} \in\left\langle\mathcal{B}_{\lambda}\right\rangle$, see [34].

The above result suggests the following algorithm. First, calculate a basis $B \in \mathbb{C}^{S \times S}$ underlying a Jordan normal form of $J(\mathbb{1})$. Afterwards, compute $\mathcal{B}_{\lambda}$ from $B \in \mathbb{C}^{S \times S}$ for all $\lambda \in \sigma(J(\mathbb{1})) \cap \mathbb{H}$. Then, any BDE space $\mathcal{U}_{\mathcal{H}}$ is spanned by vectors $w_{i}^{\lambda}$ where $w_{i}^{\lambda}=\sum_{j=1}^{d_{\lambda}} \eta_{j}^{(\lambda, i)} u_{j}^{\lambda}$ with $\eta_{j}^{(\lambda, i)} \in \mathbb{R}$ and $1 \leq i \leq k_{\lambda}$. 


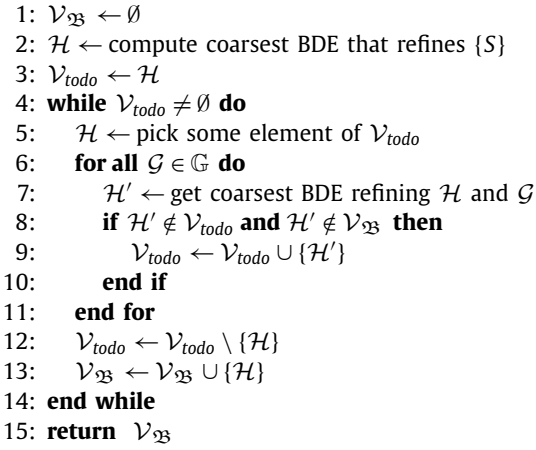

Algorithm 1. Find all BDEs of $S$ using the guiding set $\mathbb{G}=\bigcup_{\lambda} \mathbb{G}_{\lambda}$.

Example. A possible Jordan decomposition $D=B^{-1} J(\mathbb{1}) B$ of $J(\mathbb{1})$ from (3) is

$$
D=\left(\begin{array}{ccc}
0 & 0 & 0 \\
0 & \frac{3}{2}-\frac{\sqrt{3}}{2} i & 0 \\
0 & 0 & \frac{3}{2}+\frac{\sqrt{3}}{2} i
\end{array}\right), \quad B=\left(\begin{array}{ccc}
1 & -1+\sqrt{3} i & -1-\sqrt{3} i \\
1 & -1-\sqrt{3} i & -1+\sqrt{3} i \\
1 & 2 & 2
\end{array}\right)
$$

From this, we infer that $\sigma(J(\mathbb{1}))=\left\{0, \frac{3}{2}-\frac{\sqrt{3}}{2} i, \frac{3}{2}+\frac{\sqrt{3}}{2} i\right\}$. Applying the construction of Theorem 14, we obtain

$$
\mathcal{B}_{0}=\left\{(1,1,1)^{T}\right\} \quad \text { and } \quad \mathcal{B}_{\frac{3}{2}+\frac{\sqrt{3}}{2} i}=\left\{(-1,-1,2)^{T},(-\sqrt{3}, \sqrt{3}, 0)^{T}\right\}
$$

From now on, we fix a BDE partition $\mathcal{H}$ of $S$ and write $\mathcal{U}_{\mathcal{H}}=\bigoplus_{\lambda}\left\langle w_{1}^{\lambda}, \ldots, w_{k_{\lambda}}^{\lambda}\right\rangle$. Our goal is to provide an algorithm to find $\mathcal{H}$ by establishing $k_{\lambda}$ and $w_{1}^{\lambda}, \ldots, w_{k_{\lambda}}^{\lambda}$ for all $\lambda \in \sigma(J(\mathbb{1})) \cap \mathbb{H}$. To this end, we need the following.

Definition 16. Given some $v \in \mathbb{R}^{S}$, let $\mathcal{H}_{v}$ denote the finest partition of $S$ on which $v$ is constant, i.e., set $\mathcal{H}_{v}:=S / \approx_{v}$ where $x \approx_{v} y$ if and only if $v_{x}=v_{y}$. Moreover, let $\mathbb{G}(\mathcal{W}):=\left\{\mathcal{H}_{v} \mid v \in \mathcal{W}\right\}$ for any subspace $\mathcal{W} \subseteq \mathbb{R}^{S}$.

Proposition 3. It holds that $\mathcal{H}$ refines $\mathcal{H}_{v}$ for any $v \in \mathcal{U}_{\mathcal{H}}$. More generally, $\mathcal{H}$ refines $S /\left(\approx_{v_{1}} \cap \ldots \cap \approx_{v_{k}}\right)$ for any $v_{1}, \ldots, v_{k} \in \mathcal{U}_{\mathcal{H}}$. Crucially, $\mathcal{H}$ is the coarsest BDE partition that refines all $\mathcal{H}_{w_{i}^{\lambda}}$, where $\lambda \in \sigma(J(\mathbb{1})) \cap \mathbb{H}$ and $1 \leq i \leq k_{\lambda}$.

Proof. The proof is straightforward, and hence it is omitted.

Recall that Theorem 3 uses a partition refinement algorithm that takes a partition $\mathcal{G}$ as input and returns its coarsest refinement that is a BDE. Since $\mathcal{H}_{w_{i}^{\lambda}} \in \mathbb{G}\left(\left\langle u_{1}^{\lambda}, \ldots, u_{d_{\lambda}}^{\lambda}\right\rangle\right)$, the idea is to invoke our partition refinement algorithm with elements from the finite set $\bigcup_{\lambda} \mathbb{G}\left(\left\langle u_{1}^{\lambda}, \ldots, u_{d_{\lambda}}^{\lambda}\right\rangle\right)$ to perform the guided search. Our second main result is stated next and follows from Proposition 3.

Theorem 15. Given a drift $f: \mathbb{R}^{S} \rightarrow \mathbb{R}^{S}$ and the underlying set of guiding partitions $\mathbb{G}:=\bigcup_{\lambda} \mathbb{G}_{\lambda}:=\bigcup_{\lambda} \mathbb{G}\left(\left\langle u_{1}^{\lambda}, \ldots, u_{d_{\lambda}}^{\lambda}\right\rangle\right)$, Algorithm 1 computes the set of all BDE partitions $\mathfrak{B}$ of $S$ by invoking the partition refinement algorithm at most $|\mathfrak{B}| \cdot|\mathbb{G}|+1$ times.

Proof. Direct consequence of Proposition 3.

The overall complexity of Algorithm 1 also depends on the drift and on how difficult it is to calculate the sets $\mathbb{G}_{\lambda}$. For instance, if the drift arises from a system of polynomials in $|S|$ variables with degree at most two and $|R|$ denotes the number of monomials present in all polynomials (as is the case in our applications of Section 5), then the partition refinement algorithm needs $\mathcal{O}(|R| \cdot|S| \cdot \log (|S|))$ steps [24]. By using an SMT solver, the partition refinement algorithm has been extended to a richer class of drifts [14]. At the same time, however, it has been shown that the class is expressive enough to encode tautology, which is coNP-complete. Hence, efficiency holds only for subclasses.

We stress that $|\mathfrak{B}| \cdot|\mathbb{G}|+1$ is a worst case bound that is rarely attained in practice. To see why, assume for simplicity that $\mathcal{U}_{\mathcal{H}}=\left\langle w_{1}^{\lambda}, \ldots, w_{k_{\lambda}}^{\lambda}\right\rangle$ for some $\lambda \in \sigma(J(\mathbb{1})) \cap \mathbb{H}$. Then, in all examples in Section 5, we could observe that the number of subsets $W \subseteq\left\{w_{1}^{\lambda}, \ldots, w_{k_{\lambda}}^{\lambda}\right\}$ such that $\langle W\rangle$ is a BDE space was small. This implies that the coarsest BDE partition that refines $\mathcal{H}_{w_{i}^{\lambda}}$ for some $1 \leq i \leq k_{\lambda}$ is likely to be $\mathcal{H}$ itself. In other words, feeding the partition refinement algorithm with a partition underlying one single $\mathcal{H}_{w_{i}^{\lambda}}$ usually leads to a partition that refines almost all remaining partitions $\mathcal{H}_{w_{j}^{\lambda}}$, where $j \neq i$. 

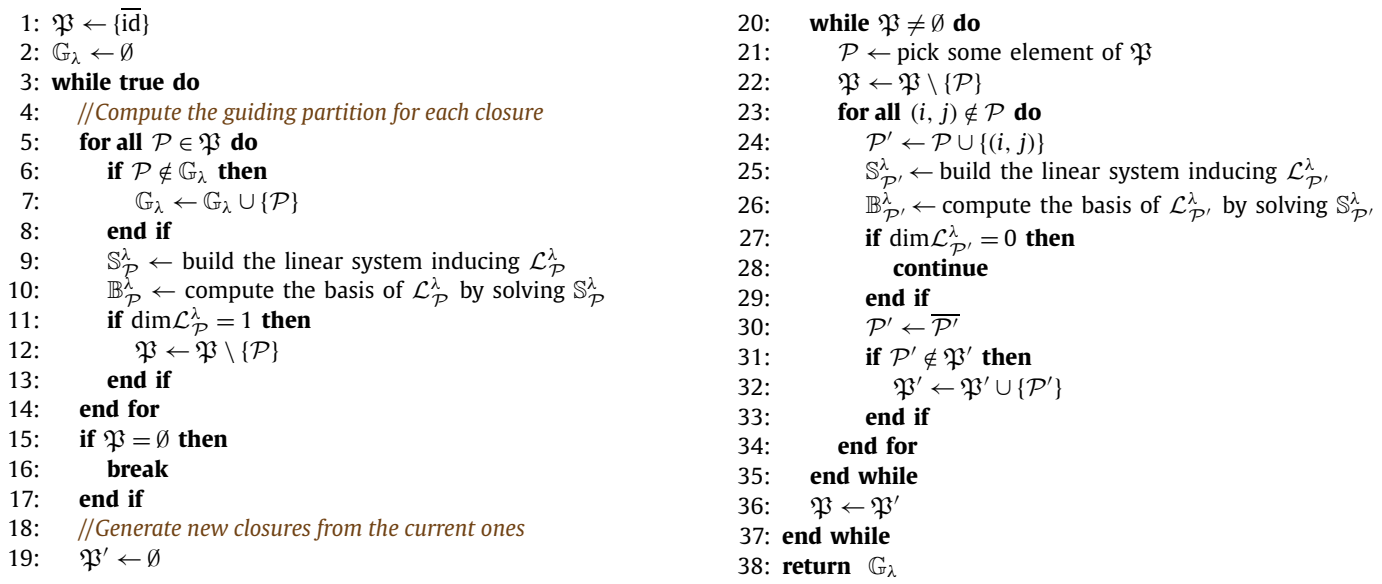

Algorithm 2. Compute the guiding set $\mathbb{G}_{\lambda}$.

We now turn to the calculation of $\mathbb{G}_{\lambda}$, with $\lambda \in \sigma(J(\mathbb{1})) \cap \mathbb{H}$. For the ease of notation, we assume in the remainder of the paragraph that $S=\{1, \ldots, n\}$ for some $n \geq 1$. The following is easily seen.

Remark 1. Theorem 14 ensures $\mathbb{G}\left(\mathcal{B}_{\lambda}\right)=\left\{\mathcal{H}_{u_{1}^{\lambda}}\right\}$ if $d_{\lambda}=1$.

In the case where $d_{\lambda}>1$, however, there are infinitely many possible directions $w_{i}^{\lambda} /\left\|w_{i}^{\lambda}\right\|$, see also discussion before Theorem 12. Note, however, that if $u \in\left\langle\mathcal{B}_{\lambda}\right\rangle$ has two coordinates, say $i$ and $j$, that coincide, then there exists an $\eta \in \mathbb{R}^{d_{\lambda}}$ such that $\sum_{l=1}^{d_{\lambda}} \eta_{l} \cdot u_{l}^{\lambda} \cdot e_{i}=\sum_{l=1}^{d_{\lambda}} \eta_{l} \cdot u_{l}^{\lambda} \cdot e_{j}$, where $e_{k}$ denotes the vector whose $k$-th coordinate is 1 and all other coordinates are zero. This motivates the following.

Definition 17. For any pair set $\mathcal{P} \subseteq\{1, \ldots, n\}^{2}$, set $\mathcal{L}_{\mathcal{P}}^{\lambda}:=\left\{\eta \in \mathbb{R}^{d_{\lambda}} \mid \forall(i, j) \in \mathcal{P}\left[\sum_{l=1}^{d_{\lambda}} \eta_{l} \cdot u_{l}^{\lambda} \cdot\left(e_{i}-e_{j}\right)=0\right]\right\}$.

That is, $\mathcal{L}_{\mathcal{P}}^{\lambda}$ denotes the coordinates $\eta$ with respect to basis $u_{1}^{\lambda}, \ldots, u_{d_{\lambda}}^{\lambda}$ that yield vectors $u \in\left\langle\mathcal{B}_{\lambda}\right\rangle$ such that the $i$-th and the $j$-th coordinates of $u$ are equal for all $(i, j) \in \mathcal{P}$. Note that any set of pairs $\mathcal{P} \subseteq S^{2}$ induces the partition $\mathcal{H}=S / \mathcal{P}^{*}$, where $\mathcal{P}^{*}$ denotes the transitive closure of $\mathcal{P}$. Thus, for $\mathcal{P}$ arbitrary, the set $\mathcal{L}_{\mathcal{P}}^{\lambda}$ is the solution space of a linear system of equations $\Theta \eta=0$ with $\Theta \in \mathbb{R}^{(n-m) \times m}$ and $m=\left|S / \mathcal{P}^{*}\right|$. We get $(n-m)$ rows because each block $\left\{i_{1}, \ldots, i_{v}\right\} \in S / \mathcal{P}^{*}$ induces $v-1$ linear equations that ensure $u\left(e_{i_{1}}-e_{i_{k}}\right)=0$ for all $2 \leq k \leq v$.

Our ultimate goal is to find all $\mathcal{H} \in \mathbb{G}_{\lambda}$ by performing a search on pair sets $\mathcal{P}$. Note that $\mathcal{P}$ can be seen as a list of constraints (namely, the pairs of coordinates that have to coincide) imposed on the linear combinations of $u_{1}^{\lambda}, \ldots, u_{d_{\lambda}}^{\lambda}$. In particular, by adding additional pairs to $\mathcal{P}$, the dimension of $\mathcal{L}_{\mathcal{P}}^{\lambda}$ can either stay the same or become smaller. (The dimension can stay the same, for instance, if $u\left(e_{i}-e_{j}\right)=0$ whenever $u\left(e_{j}-e_{k}\right)=0$. In such case, one gets $\mathcal{L}_{\{(i, j)\}}^{\lambda}=$ $\mathcal{L}_{\{(i, j),(j, k)\}}^{\lambda}=\mathcal{L}_{\{(j, k)\}}^{\lambda}$, meaning that adding $(j, k)$ to $\{(i, j)\}$ (or $(i, j)$ to $\left.\{(j, k)\}\right)$ does not reduce the dimension.)

With this in mind, we make the following pivotal observation. Define for any set of pairs $\mathcal{P}$ the underlying closure as $\overline{\mathcal{P}}:=\left\{(i, j) \mid \mathcal{L}_{\mathcal{P} \cup\{(i, j)\}}=\mathcal{L}_{\mathcal{P}}\right\}$. It is not hard to see that $\overline{\mathcal{P}}$ is the largest equivalence relation such that $\sum_{l=1}^{d_{\lambda}} \eta_{l} \cdot u_{l}^{\lambda} \cdot\left(e_{i}-e_{j}\right)=$ 0 for all $(i, j) \in \overline{\mathcal{P}}$ and $\eta \in \mathcal{L}_{\mathcal{P}}$. Consequently, the solution space $\mathcal{L}_{\mathcal{P}}$ corresponds to the partition $S / \overline{\mathcal{P}}$ in $\mathbb{G}_{\lambda}$. Note also that $\mathcal{L}_{\overline{\mathrm{id}}}=\mathbb{R}^{d_{\lambda}}$ and that $\operatorname{dim} \mathcal{L}_{\overline{\mathcal{P}} \cup\{(i, j)\}}<\operatorname{dim} \mathcal{L}_{\overline{\mathcal{P}}}=\operatorname{dim} \mathcal{L}_{\mathcal{P}}$ for any pair set $\mathcal{P}$ and $(i, j) \notin \overline{\mathcal{P}}$. Consequently, by starting with the closure $\overline{\mathrm{id}}$, the set of all closures can be obtained recursively by visiting, for any computed closure $\mathcal{P}$, its underlying closures $\overline{\mathcal{P} \cup\{(i, j)\}}$, where $(i, j) \notin \mathcal{P}$.

The above observation is formalized in Algorithm 2. There, $\mathfrak{P}$ contains the closures that are processed in the current iteration of the main while loop in line 3 . Since id $=\{(i, i) \mid 1 \leq i \leq n\}$ corresponds to the maximal solution space $\mathbb{R}^{d_{\lambda}}$ and each iteration of the main while loop seeks to reduce the maximal dimension present in $\mathfrak{P}$, we initialize it with the closure of id. We now discuss the body of the main while loop. Lines 5-14 compute for each closure $\mathcal{P} \in \mathfrak{P}$ the underlying guiding partition $\{1, \ldots, n\} / \mathcal{P}$. In the case the solution space underlying a closure $\mathcal{P}$ has dimension one, adding any further pair $(i, j) \notin \mathcal{P}$ will lead to the trivial solution space $\mathcal{L}_{\mathcal{P} \cup\{(i, j)\}}=\emptyset$. Consequently, we can remove $\mathcal{P}$ from $\mathfrak{P}$. Lines $20-35$ compute for each $\mathcal{P}^{\prime} \in\{\mathcal{P} \cup\{(i, j)\} \mid \mathcal{P} \in \mathfrak{P},(i, j) \notin \mathcal{P}\}$ the underlying closure $\overline{\mathcal{P}^{\prime}}$ and add it to $\mathfrak{P}^{\prime}$. Crucially, line 31 ensures that no closure is added more than once to $\mathfrak{P}^{\prime}$. Although this check does not improve the worst case bounds, it allows for a substantial speed-up in practice.

Before discussing the correctness and the running time of the algorithm, we discuss it on an example. 
Example. Let us apply Algorithm 2 to the bases given in (4). The case $\mathcal{B}_{0}=\left\{(1,1,1)^{T}\right\}$ yields $\mathbb{G}_{0}=\{\{\{1,2,3\}\}\}$, so let us focus on $\mathcal{B}_{\lambda}=\left\{(-1,-1,2)^{T},(-\sqrt{3}, \sqrt{3}, 0)^{T}\right\}$ with $\lambda=\frac{3}{2}+\frac{\sqrt{3}}{2} i$. We first calculate $\mathcal{L}_{\{(1,2)\}}^{\lambda}, \mathcal{L}_{\{(1,3)\}}^{\lambda}$ and $\mathcal{L}_{\{(2,3)\}}^{\lambda}$. By definition, $\left(\eta_{1}, \eta_{2}\right) \in \mathcal{L}_{\{(1,2)\}}^{\lambda}$ whenever

$$
0=\left(\eta_{1}(-1,-1,2)^{T}+\eta_{2}(-\sqrt{3}, \sqrt{3}, 0)^{T}\right)\left(e_{1}-e_{2}\right)
$$

which is equivalent to $-\eta_{1}-\sqrt{3} \eta_{2}=-\eta_{1}+\sqrt{3} \eta_{2}$. That is, $\mathcal{L}_{\{(1,2)\}}^{\lambda}=\left\{\left(\eta_{1}, \eta_{2}\right) \mid \eta_{2}=0\right\}$. Further, we infer that $\left(\eta_{1}, \eta_{2}\right) \in \mathcal{L}_{\{(1,3)\}}^{\lambda}$ if and only if $-\eta_{1}-\sqrt{3} \eta_{2}=2 \eta_{1}$, yielding $\mathcal{L}_{\{(1,3)\}}^{\lambda}=\left\{\left(\eta_{1}, \eta_{2}\right) \mid \eta_{2}=-\sqrt{3} \eta_{1}\right\}$. In a similar fashion one obtains $\mathcal{L}_{\{(2,3)\}}^{\lambda}=$ $\left\{\left(\eta_{1}, \eta_{2}\right) \mid \eta_{2}=\sqrt{3} \eta_{1}\right\}$. The vectors induced by $\mathcal{L}_{\{(1,2)\}}^{\lambda}, \mathcal{L}_{\{(1,3)\}}^{\lambda}$ and $\mathcal{L}_{\{(2,3)\}}^{\lambda}$ are thus respectively given by:

$$
\begin{aligned}
\left(\eta_{1}, \eta_{1}, 2 \eta_{1}\right)^{T} & =\eta_{1}(-1,-1,2)^{T}+0 \cdot(-\sqrt{3}, \sqrt{3}, 0)^{T} \\
\left(2 \eta_{1},-4 \eta_{1}, 2 \eta_{1}\right)^{T} & =\eta_{1}(-1,-1,2)^{T}-\sqrt{3} \eta_{1}(-\sqrt{3}, \sqrt{3}, 0)^{T} \\
\left(-4 \eta_{1}, 2 \eta_{1}, 2 \eta_{1}\right)^{T} & =\eta_{1}(-1,-1,2)^{T}+\sqrt{3} \eta_{1}(-\sqrt{3}, \sqrt{3}, 0)^{T},
\end{aligned}
$$

where $\eta_{1} \in \mathbb{R}$. Since $\mathcal{L}_{\{(1,2)\}}^{\lambda}, \mathcal{L}_{\{(1,3)\}}^{\lambda}$ and $\mathcal{L}_{\{(2,3)\}}^{\lambda}$ are pairwise different subspaces of $\mathbb{R}^{2}$, it holds that $\overline{\mathrm{id}}=\mathrm{id}$ and the (trivial) guiding partition $\{\{1\},\{2\},\{3\}\}$ is added to $\mathbb{G}_{\lambda}$ by lines $5-14$. Afterwards, lines $20-35$ set $\mathfrak{P}^{\prime}$ to $\{\operatorname{id} \cup\{(1,2)\}$, id $\cup\{(1,3)\}$, id $\cup$ $\{(2,3)\}\}$ and the solutions spaces $\mathcal{L}_{\{(1,2)\}}^{\lambda}, \mathcal{L}_{\{(1,3)\}}^{\lambda}$ and $\mathcal{L}_{\{(2,3)\}}^{\lambda}$ are computed. The first iteration finishes by giving $\mathfrak{P}$ the value of $\mathfrak{P}^{\prime}$. During the second iteration, lines 5-14 add $\{\{1,2\},\{3\}\},\{\{1,3\},\{2\}\},\{\{1\},\{2,3\}\}$ to $\mathbb{G}_{\lambda}$ while emptying $\mathfrak{P}$. This is because $\mathcal{L}_{\{(1,2)\}}^{\lambda}, \mathcal{L}_{\{(1,3)\}}^{\lambda}$ and $\mathcal{L}_{\{(2,3)\}}^{\lambda}$ are all of dimension one. The algorithm terminates then in the second iteration with $\mathbb{G}_{\lambda}=\{\{\{1\},\{2\},\{3\}\},\{\{1,2\},\{3\}\},\{\{1,3\},\{2\}\},\{\{1\},\{2,3\}\}\}$.

The following crucial result can be shown.

Theorem 16. If $d_{\lambda}=1$, then $\mathbb{G}_{\lambda}=\left\{u_{1}^{\lambda}\right\}$. Instead, if $d_{\lambda}>1$, Algorithm 2 computes $\mathbb{G}_{\lambda}$ in at most $\mathcal{O}\left(n^{2 d_{\lambda}+4}\right)$ steps.

Before giving the proof, we again stress that such bounds are due to the pessimistic assumption that lines 20-35 lead to pairwise different closures. However, the algorithm could be successfully applied to all models in Section 5 , because each iteration had a substantial number of closures $\mathcal{P}, \mathcal{P}^{\prime} \in \mathfrak{P}$ and pairs $(i, j) \notin \mathcal{P},\left(i^{\prime}, j^{\prime}\right) \notin \mathcal{P}^{\prime}$ such that $\overline{\mathcal{P} \cup\{(i, j)\}}=$ $\overline{\mathcal{P}^{\prime} \cup\left\{\left(i^{\prime}, j^{\prime}\right)\right\}}$. In particular, in the case of a highly symmetric network with $n=12$ and $\max _{\lambda} d_{\lambda}=7$, we were able to calculate all BDE partitions in around $40 \mathrm{~min}$ on a $2.6 \mathrm{GHz}$ Intel Core i5 machine with 4GB of RAM.

We also argue that, although $d_{\lambda}$ can be equal to $n$, usually $\max _{\lambda} d_{\lambda} \ll n$. In fact, it is well-known that the set of $n \times n$ matrices that have $n$ pairwise different eigenvalues (which suffices $\max _{\lambda} d_{\lambda} \leq 2$ ) is dense in $\mathbb{R}^{n \times n}$.

Remark 2. Algorithm 2 allows for a bounded computation if the additional break condition $\left(\begin{array}{l}n \\ 2\end{array}\right) \cdot|\mathfrak{P}|>$ bound is added in line 15. Although a bounded computation may fail to find all BDE partitions, the number of missed partitions can be expected to be small if bound is of decent size because, as pointed out in the discussion after Theorem 15, Algorithm 1 usually needs only a subset of the guiding set in order to find all BDE partitions. Moreover, note that Algorithm 1 always returns a set of BDE partitions, meaning that the bounded computation is sound.

Proof. The correctness of the algorithm follows from the discussion after Definition 17. In the following, all line numbers refer to Algorithm 2. Let $\mathfrak{P}_{v}$ be the content of $\mathfrak{P}$ at the beginning of iteration $v$ of the while loop in line 3 , where the first iteration has index $v=0$. By construction, Algorithm 2 ensures that $\operatorname{dim} \mathcal{L}_{\mathcal{P}} \leq d_{\lambda}-v$ for any $\mathcal{P} \in \mathfrak{P}_{v}$. Since closures whose solution space has dimension one are removed in lines $5-14$, this ensures that there are at most $d_{\lambda}$ iterations of the main while loop. Moreover, we note that lines 20-35 increase the number of pair sets at most by the factor $\left(\begin{array}{l}n \\ 2\end{array}\right) \leq n^{2}$, hence $\left|\mathfrak{P}_{v}\right| \leq\left|\mathfrak{P}_{v-1}\right| \cdot n^{2}$ for all $v \geq 1$, thus yielding $\left|\mathfrak{P}_{v}\right| \leq\left(n^{2}\right)^{v}$ for all $v \geq 0$. By combining both statements, we infer that $\mathfrak{P}$ cannot exceed the size of $\left|\mathfrak{P}_{d_{\lambda}-1}\right| \leq\left(n^{2}\right)^{d_{\lambda}-1}=n^{2\left(d_{\lambda}-1\right)}$. We now take a closer look at the algorithm. Any closure $\mathcal{P}$ is stored in terms of the corresponding partition $\{1, \ldots, n\} / \mathcal{P}$ and each partition is encoded by a row vector $p \in \mathbb{R}^{1 \times n}$ where $p(i)$ denotes the smallest index of the block to which $i$ belongs (e.g., the partition $\{\{1,3\},\{2,4\}$, $\{5\}\}$ is encoded by the row vector $(1,2,1,2,5)$ ). With this, lines $9,10,24,25$ and 26 can be solved in $\mathcal{O}\left(n^{3}\right)$ steps. The computation in line 30 is accomplished in the following way. With $v_{1}, \ldots, v_{d}$ being the vectors of $\mathbb{B}_{\mathcal{P}^{\prime}}^{\lambda}$, we first calculate $\mathcal{H}_{v_{1}}, \ldots, \mathcal{H}_{v_{d}}$. Afterwards, we compute the coarsest partition that refines all $\mathcal{H}_{v_{1}}, \ldots, \mathcal{H}_{v_{d}}$. Since $d \leq n$, line 30 thus needs at most $\mathcal{O}\left(n^{4}\right)$ steps. We further note that the number of entries in $\mathfrak{P}, \mathfrak{P}^{\prime}$ and $\mathbb{G}_{\lambda}$ is bounded by $\sum_{i=0}^{v}\left(n^{2}\right)^{i} \leq\left(n^{2}\right)^{v+1}$ in the $v$-th iteration. Hence, if $\mathfrak{P}, \mathfrak{P}^{\prime}$ and $\mathbb{G}_{\lambda}$ are implemented as red-black trees, the underlying methods add, remove and in will cost at most $\mathcal{O}\left(\log \left(\left(n^{2}\right)^{v+1}\right) \cdot n\right) \leq \mathcal{O}\left(n^{2} \cdot \log (n)\right)$. (The multiplication by $n$ is due to the sorting with respect to partitions.)

This shows that the $v$-th iteration of the main while loop costs at most $\left(n^{2}\right)^{v} \cdot \mathcal{O}\left(n^{4}\right)$ steps. Since lines 20-35 are not invoked in the last iteration and there are at most $d_{\lambda}$ iterations, the total number of operations is at most $\sum_{0 \leq \nu \leq d_{\lambda}-1}\left(n^{2}\right)^{v}$. $\mathcal{O}\left(n^{4}\right) \leq \mathcal{O}\left(n^{2 d_{\lambda}+4}\right)$. 
The computation of a Jordan decomposition of a matrix $A \in \mathbb{R}^{n \times n}$ takes $\mathcal{O}\left(n^{3}\right)$ steps. Instead, $J(\mathbb{1})$ can be calculated in polynomial time if the drift $f$ is such that each $f_{i}: \mathbb{R}^{n} \rightarrow \mathbb{R}$ is a polynomial in several variables. Consequently, Algorithm 1 runs in polynomial time for polynomial systems.

Calculating all emulations. With Algorithm 1 it is easy to decide whether two ODE systems are related by means of an emulation. Fix a source drift $f: \mathbb{R}^{S} \rightarrow \mathbb{R}^{S}$ and a target drift $\hat{f}: \mathbb{R}^{\hat{S}} \rightarrow \mathbb{R}^{\hat{S}}$ such that $S \cap \hat{S}=\emptyset$. Since one can always rename variables, we can make this assumption without loss of generality.

Recall that Proposition 1 ensures that $\mu: S \rightarrow \hat{S}$ is an emulation if and only if $\left\{\mu^{-1}(\hat{x}) \cup\{\hat{x}\} \mid \hat{x} \in \hat{S}\right\}$ is a BDE partition of $S \cup \hat{S}$. Thus, if we apply Algorithm 1 to the union drift $g: \mathbb{R}^{S \cup \hat{S}} \rightarrow \mathbb{R}^{S \cup \hat{S}}$ where $g_{x}(w)=f_{x}\left(w_{\mid S}\right)$ and $g_{\hat{x}}(w)=\hat{f}_{\hat{x}}\left(w_{\mid \hat{S}}\right)$ for all $w \in \mathbb{R}^{S \cup \hat{S}}$, all we have to do is to check whether there exist BDE partitions $\mathcal{H}$ of $S \cup \hat{S}$ that satisfy $|\mathcal{H}|=|\hat{S}|$ and $|H \cap S| \geq|H \cap \hat{S}|=1$ for all $H \in \mathcal{H}$.

Another approach is to apply Algorithm 1 to the source drift $f: \mathbb{R}^{S} \rightarrow \mathbb{R}^{S}$. In the case there are no BDE partitions $\mathcal{H}$ of $S$ that have exactly $|\hat{S}|$ blocks, the source and the target drifts cannot be related to each other. In the case there are, one has to decide for each $\mathcal{H}$ of $S$ that satisfies $|\mathcal{H}|=|\hat{S}|$ whether the target drift is isomorphic to the aggregated drift underlying $f$ and $\mathcal{H}$. That is, one has to decide whether there exists a $\mu: S \rightarrow S$ with $\mathcal{H}=\left\{\mu^{-1}(x) \mid x \in \mu(S)\right\}$ and a renaming $\eta: \mu(S) \rightarrow \hat{S}$ such that $\hat{f}_{\hat{x}}(\hat{v})=f_{\eta^{-1}(\hat{x})}(\hat{v} \circ \eta \circ \mu)$ for all $\hat{v} \in \mathbb{R}^{\hat{S}}$ and $\hat{x} \in \hat{S}$. This has the advantage that Algorithm 1 considers the target drift $f$ instead of the union drift $g$, at the expense of deciding whether two drifts are isomorphic. We use this approach in Section 5 because all target networks considered there are of moderate size.

\section{Applications}

This section applies our approach to networks from the literature. Experiments are replicable using our prototype CAGE, which extends ERODE [25], as explained in http://sysma.imtlucca.it/tools/erode/cage/. Inputs consist of mass-action CRNs or polynomial ODEs. ERODE acts as an integrated development environment for mass-action CRNs or polynomial ODEs, for which it automatically generates MATLAB scripts implementing Algorithms 1 and 2. Jacobian matrices are computed syntactically as in [44], while the coarsest BDEs are computed within MATLAB by invoking the corresponding command on a library version of ERODE.

Influence networks. Sections 5.1 and 5.2 consider a class of CRNs known as influence networks (e.g., [10]). A nice property of influence networks is that they allow for a compact representation, depicted in Figs. 2, 4, and 5. For example, the influence network in Fig. 2 (left) corresponds to a CRN with six species, $x a_{0}, x a_{1}, x a_{2}, y a_{0}, y a_{1}, y a_{2}$, and four reactions:

$$
\begin{aligned}
x a_{0}+y a_{0} & \rightarrow{ }^{\alpha_{1}} y a_{0}+x a_{1}, \\
y a_{0}+x a_{1} & \rightarrow{ }^{\alpha_{2}} y a_{0}+x a_{2}, \\
x_{0}+y_{2} & \rightarrow{ }^{\alpha_{3}} x_{0}+y_{1}, \\
x_{0}+y_{1} & \rightarrow{ }^{\alpha_{4}} x_{0}+y_{0}
\end{aligned}
$$

Each node (e.g., $x a$ and $y a$ ) corresponds to three chemical species (e.g., $x a_{0}, x a_{1}, x a_{2}$, and $y a_{0}, y a_{1}, y a_{2}$ ) and at most four chemical reactions, depending on how nodes are connected. Nodes are connected via influence edges, denoting inhibition or promotion influences among nodes. In particular, each node can have four kinds of connections: i) high output (denoted by a solid line, e.g., the top of the left edge of Fig. 2), representing an influence exerted by the species with subscript 0; ii) low output (denoted by a dashed line, e.g., the bottom edge of node $r$ in Fig. 4 (d)), representing an influence exerted by the species with subscript 2; iii) activation input (circle, e.g., the bottom of the left edge of Fig. 2); and iv) inhibition input (bar, e.g., the top of the right edge of node xa of Fig. 2). Species with index 1 introduce nonlinearity in transitions [10] and are never otherwise connected to the network. If inh and act are the inhibitor and activation input species for a node $x a$, respectively, then $x a$ is associated with the following reactions:

$$
\begin{aligned}
& x a_{0}+i n h \rightarrow^{\alpha_{01}} i n h+x a_{1}, \\
& x a_{1}+i n h \rightarrow^{\alpha_{12}} i n h+x a_{2}, \\
& x a_{2}+a c t \rightarrow^{\alpha_{21}} a c t+x a_{1}, \\
& x a_{1}+a c t \rightarrow^{\alpha_{10}} a c t+x a_{0},
\end{aligned}
$$

where $\alpha_{01}, \alpha_{12}, \alpha_{21}, \alpha_{10}$ are given rate coefficients of node $x a$. An influence network is called unimodal if all species are activated or inhibited by at most one species. In other words, in unimodal influence networks each species can have at most one inhibitor and one activator. 

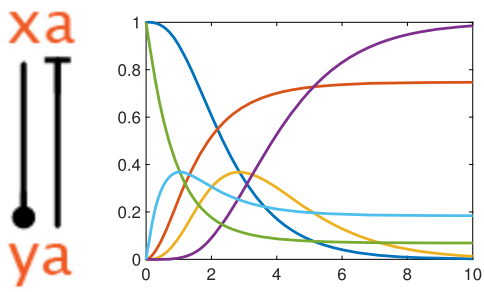

(a) $N=1$
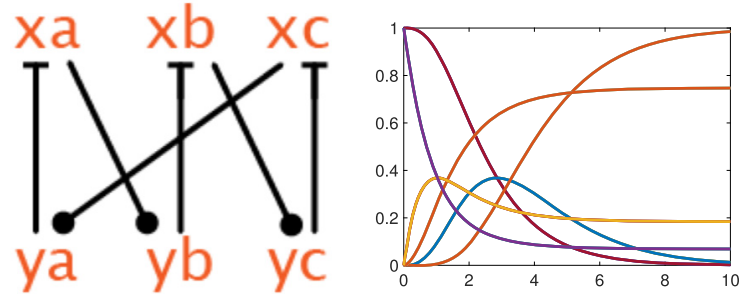

(b) $N=3$

Fig. 2. Simple (a) and randomly replicated influence network (b), paired with corresponding ODE solutions (where any rate is set to 1 ).

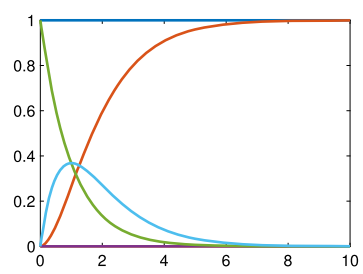

(a) $N=1[y a]$

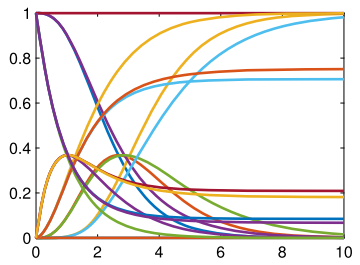

(e) $N=3[y c]$

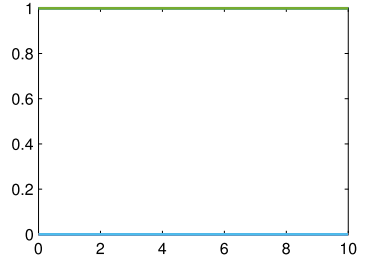

(b) $N=1[x a]$

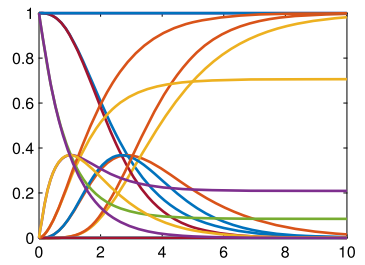

(f) $N=3[x a]$

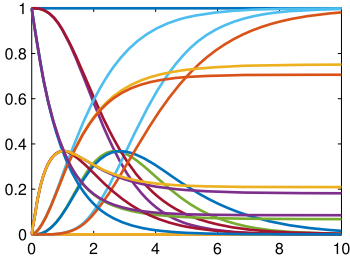

(c) $N=3[y a]$

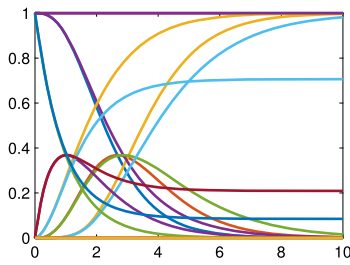

(g) $N=3[x b]$

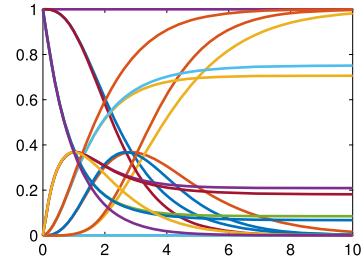

(d) $N=3[y b]$

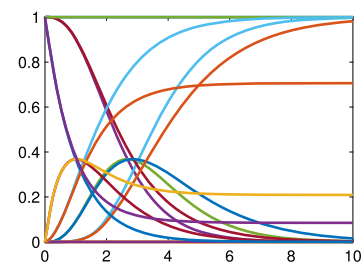

(h) $N=3[x c]$

Fig. 3. ODE solutions of variants of the networks from Fig. 2 obtained by removing the influence exerted by the specified node.

\subsection{Randomly replicated influence networks}

It is well known that using larger networks instead of smaller ones of equal functionality is beneficial for enhanced stability with respect to stochastic noise $[11,12]$. We hereby instantiate this to the case where the equivalent functionality is captured by an emulation. This is done using the synthetic influence networks depicted in Fig. 2. The network in Fig. 2(a) consists of nodes $x a$ and $y a$, where the high output of $x a$ promotes $y a$, while the high output of $y a$ inhibits $x a$. Instead, Fig. 2(b) depicts a variant of Fig. 2(a) with three replicas of each original node, where the promotion/inhibition relations have been randomly generated under the constraint that each $x_{-}$node has one outgoing promotion and one incoming inhibition arc, and each $y_{-}$node has one outgoing inhibition and one incoming promotion arc. Fig. 2 also plots the ODE solutions of the two networks computed setting all rates to 1 , for initial conditions equal to 1 for the eight species $x \_0$ and $y \_2$, and equal to 0 for the others.

Interestingly, it can be shown that any network generated as in Fig. 2(b) emulates the one in Fig. 2(a). Indeed, as exemplified by the plots in Fig. 2, CAGE is able to discover the following BDE partition on the union of the networks in Fig. 2: $\left\{\left\{X a_{0}, X b_{0}, X c_{0}, x a_{0}\right\},\left\{X a_{1}, X b_{1}, X c_{1}, x a_{1}\right\},\left\{X a_{2}, X b_{2}, X c_{2}, x a_{2}\right\},\left\{Y a_{0}, Y b_{0}, Y c_{0}, y a_{0}\right\},\left\{Y a_{1}, Y b_{1}, Y c_{1}, y a_{1}\right\}\right.$, $\left.\left\{Y a_{2}, Y b_{2}, Y c_{2}, y a_{2}\right\}\right\}$, where we indicate the species of Fig. 2(b) with capital letters.

Notably, the replication of nodes leads to more robust implementations of the original dynamics. This is exemplified in Fig. 3: Figs. 3(a)-(b) plot the ODE solutions of variants of Fig. 2(a) obtained by removing the influence originating from the nodes in square brackets. Figs. 3(c)-(h) are similar, but refer to variants of Fig. 2(b). We note that the perturbations introduced in the network in Fig. 2(a) destroy its dynamics, while the effect is weaker for the one in Fig. 2(b).

The next section provides further examples of redundant dynamics arising in evolutionary biology.

\subsection{Evolutionary biology}

Fig. 4 depicts the unimodal influence networks studied in [10]. The AM network [45] models a cell cycle switch that is needed to avoid genetic instability during replication. Instead, MI, SI, and QI are different examples of mutual inhibition networks, a mechanism present in many biological networks (e.g. [46,47]). The networks CCR, GW, and NCC are more 


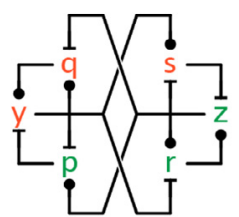

(a) $\mathrm{NCC}$

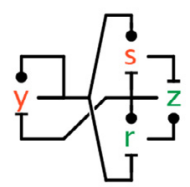

(b) GW

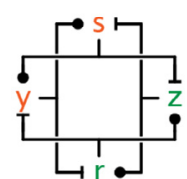

(c) QI

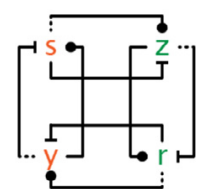

(d) $\mathrm{DN}$

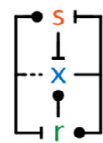

(e) $\mathrm{CCR}$

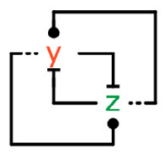

(f) SI

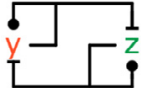

(g) MI

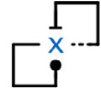

(h) AM

Fig. 4. Unimodal influence networks from [10].

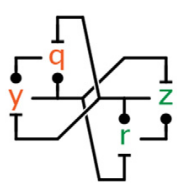

(a) GW'

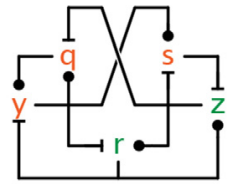

(b) NCC'

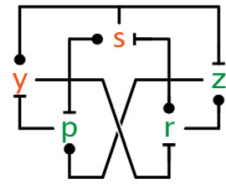

(c) NCC"

Fig. 5. Unimodal influence networks not studied in [10].

efficient variants of the G2-M cell cycle switch [48]. Finally, the network DN is a schematic Delta-Notch configuration between two neighboring cells (represented by the pairs of nodes $s, z$, and $y, r$, respectively) [49].

One of the main results of [10] was to show, by hand, that all networks in Fig. 4 emulate AM, which essentially means that they implement more complex versions of a cell cycle switch. In [10], Cardelli gives an evolutionary interpretation to the found emulations, arguing that the complex networks may descend from AM conservatively [12]. In addition, some of the networks in Fig. 4 have been studied in [11,12] where it has been observed that larger networks are less affected by stochastic noise than the underlying emulation-equivalent smaller networks.

By invoking Algorithm 1, we were able to automatically rediscover the emulations from [10] (where any rate was set to 1). Moreover, we applied our algorithm also to the networks in Fig. 5, which are further possible evolutionary transitions between cell cycle switches that were not covered in [10]. This provided us with a computer-aided proof of the fact that also those networks emulate AM. Indeed, we found the following union BDE partitions:

$$
\begin{aligned}
\mathcal{H}_{\text {GW' } \cup \text { AM }} & =\left\{\left\{x_{0}, q_{0}, r_{2}, y_{0}, z_{2}\right\},\left\{x_{1}, q_{1}, r_{1}, y_{1}, z_{1}\right\},\left\{x_{2}, q_{2}, r_{0}, y_{2}, z_{0}\right\}\right\}, \\
\mathcal{H}_{\mathrm{NCC} \cup \mathrm{AM}} & =\left\{\left\{x_{0}, q_{2}, r_{0}, s_{2}, y_{2}, z_{0}\right\},\left\{x_{1}, q_{1}, r_{1}, s_{1}, y_{1}, z_{1}\right\},\left\{x_{2}, q_{0}, r_{2}, s_{0}, y_{0}, z_{2}\right\}\right\}, \\
\mathcal{H}_{\mathrm{NCC} \cup \mathrm{AM}} & =\left\{\left\{x_{0}, p_{2}, r_{2}, s_{0}, y_{0}, z_{2}\right\},\left\{x_{1}, p_{1}, r_{1}, s_{1}, y_{1}, z_{1}\right\},\left\{x_{2}, p_{0}, r_{0}, s_{2}, y_{2}, z_{0}\right\}\right\} .
\end{aligned}
$$

The algorithm can also be used to verify that no emulation can relate two unimodal influence networks. In such a case, one can infer that two networks do not share certain biological properties, such as switches. For instance we verified that NCC does not emulate GW in the case where all rate coefficients are set to one, while they both emulate AM.

As with all quantitative notions of model comparison, emulation is sensitive to rate parameters. We now study how this dependency can be loosened for the case of unimodal influence networks. First we show that the assumptions made in Theorem 9 can be dropped, i.e., the change of rates theorem from [10] carries over to flux morphisms.

Theorem 17. Fix a source $C R N(S, R)$, a target unimodal influence network $(\hat{S}, \hat{R})$ and let $(\mu, \sigma) \in(S \rightarrow \hat{S}) \times(R / \sim \hookrightarrow \hat{R} / \sim)$ be a flux morphism. Then, for any change of rates $\hat{\imath}: \hat{R} \rightarrow \hat{R}^{\prime}$, there exists a change of rates $\iota: R \rightarrow R^{\prime}$ such that $\left(\mu, \sigma^{\prime}\right):\left(S, R^{\prime}\right) \hookrightarrow\left(\hat{S}, \hat{R}^{\prime}\right)$, where $\sigma^{\prime}$ is induced by $\mu: S \rightarrow \hat{S}$ and condition (2), is a flux morphism.

Proof. Observe that the proof of Theorem 9 relies on the fact that

$$
\sum_{e \in \sigma^{-1}(\hat{e})} \sum_{r \in e} \underbrace{\left(\pi_{r}(x)-\rho_{r}(x)\right) \cdot \alpha_{r}}_{=\phi(x, r)}=\sum_{\hat{r} \in \hat{e}} \underbrace{\left(\hat{\pi}_{\hat{r}}(\mu(x))-\hat{\rho}_{\hat{r}}(\mu(x))\right) \cdot \hat{\alpha}_{\hat{r}}}_{=\phi(\mu(x), \hat{r})}
$$

simplifies, under the assumption of $\sim$-uniformity, to

$$
\sum_{e \in \sigma^{-1}(\hat{e})} \sum_{r \in e}\left(\pi_{r}(x)-\rho_{r}(x)\right) \cdot \alpha_{r}=\sum_{\hat{r} \in \hat{e}}\left(\hat{\pi}_{\hat{r}}(\mu(x))-\hat{\rho}_{\hat{r}}(\mu(x))\right) \cdot \hat{\alpha}_{\hat{e}}
$$

In particular, note that the proof of Theorem 9 goes also through if, for all $\hat{e} \in \hat{R} / \sim$, it holds that:

- either $\left(\hat{\pi}_{\hat{r}}(\mu(x))-\hat{\rho}_{\hat{r}}(\mu(x)) \leq 0\right.$ for all $\hat{r} \in \hat{e}$ and $x \in S$,

- or $\left(\hat{\pi}_{\hat{r}}(\mu(x))-\hat{\rho}_{\hat{r}}(\mu(x))\right) \geq 0$ for all $\hat{r} \in \hat{e}$ and $x \in S$. 
Observe that since $(\hat{S}, \hat{R})$ is a unimodal influence network, $\hat{S}=\bigcup_{j \in I}\left\{\hat{x}_{0}^{j}, \hat{x}_{1}^{j}, \hat{x}_{2}^{j}\right\}$ for some index set $I$. Fix an arbitrary $\hat{e} \in \hat{R} / \sim$ and $x \in S$. Let us assume that $\mu(x)=\hat{x}_{0}^{j}$ for some $j \in I$. We note that $\left(\hat{\pi}_{\hat{r}}(\mu(x))-\hat{\rho}_{\hat{r}}(\mu(x))=0\right.$ for all reactions $\hat{r}$ in which $\hat{x}_{0}^{j}$ acts solely as an activator or inhibitor. Consequently, the only two reactions in $\hat{R}$ that may lead to $\left(\hat{\pi}_{\hat{r}}(\mu(x))-\right.$ $\left.\hat{\rho}_{\hat{r}}(\mu(x))\right) \neq 0$ are $\hat{x}_{0}^{j}+i \rightarrow^{\alpha_{01}} i+\hat{x}_{1}^{j}$ and $\hat{x}_{1}^{j}+a \rightarrow^{\alpha_{10}} a+\hat{x}_{0}^{j}$ (where $\hat{x}_{0}^{j} \in\{i, a\}$ is possible as well). Since activators and inhibitors can be only low and high species, the aforementioned reactions cannot belong to $\hat{e}$ at the same time, meaning $\hat{e}$ satisfies the desired condition if $\mu(x)=\hat{x}_{0}^{j}$ for some $j \in I$.

The case where $\mu(x)=\hat{x}_{2}^{j}$ for some $j \in I$ is similar, so let us assume that $\mu(x)=\hat{x}_{1}^{j}$ for some $j \in I$. Since $(\hat{S}, \hat{R})$ is unimodal, it cannot act as an activator or inhibitor, meaning that the only reactions in which $\hat{x}_{1}^{j}$ appears are:

$$
\hat{x}_{0}^{j}+i \rightarrow^{\alpha_{01}} i+\hat{x}_{1}^{j}, \quad \hat{x}_{1}^{j}+i \rightarrow^{\alpha_{12}} i+\hat{x}_{2}^{j}, \quad \hat{x}_{2}^{j}+a \rightarrow^{\alpha_{21}} a+\hat{x}_{1}^{j}, \quad \hat{x}_{1}^{j}+a \rightarrow^{\alpha_{10}} a+\hat{x}_{0}^{j} .
$$

Let us first assume that $\hat{x}_{0}^{j}+i \rightarrow^{\alpha_{01}} i+\hat{x}_{1}^{j}=\hat{r} \in \hat{e}$. Then, since activators and inhibitors can be only low and high species, we have $\hat{x}_{1}^{j}+i \rightarrow^{\alpha_{12}} i+\hat{x}_{2}^{j} \notin \hat{e}$ and $\hat{x}_{1}^{j}+a \rightarrow^{\alpha_{10}} a+\hat{x}_{0}^{j} \notin \hat{e}$. Instead, if $i=\hat{x}_{2}^{j}$ and $a=\hat{x}_{0}^{j}, \hat{x}_{2}^{j}+a \rightarrow^{\alpha_{21}} a+\hat{x}_{1}^{j} \in \hat{e}$, but this is fine because $\hat{x}_{1}^{j}$ has, similarly to $\hat{r}$, a positive stoichiometry in this reaction. Similar arguments apply also to the other three cases, thus showing the claim.

Dually, it is interesting to ask whether the absence of emulations holds true for all possible rates. Unfortunately, it is not clear how to lift Algorithm 2 in the case when $J(\mathbb{1})$ has variables as entries because this leads to parametric bases $\mathcal{B}_{\lambda}$. Another problem is that, in general, the eigenvalues of a matrix with parameters cannot be expressed in terms of formulae because of Abel's impossibility theorem. Instead, we tackle this problem in a different way.

Definition 18. Let $(S, R)$ and $(\hat{S}, \hat{R})$ be two unimodal influence networks. An emulation $\mu: S \rightarrow \hat{S}$ is triplet preserving if for any triplet $x_{0}, x_{1}, x_{2}$ of $(S, R)$ there exists a triplet $\hat{x}_{0}, \hat{x}_{1}, \hat{x}_{2}$ of $(\hat{S}, \hat{R})$ such that $\mu\left(x_{1}\right)=\hat{x}_{1}$ and $\left\{\mu\left(x_{0}\right), \mu\left(x_{2}\right)\right\}=\left\{\hat{x}_{0}, \hat{x}_{2}\right\}$.

In the case of unimodal influence networks, only triplet preserving emulations reveal biologically meaningful relations (since subscript-one species are intermediate). The next result is based on Theorem 17 and allows one to argue about families of networks by considering a single pair of networks with unit rates. For instance, since NCC does not emulate GW when all rates are set to one, the following theorem ensures that there exists no triplet preserving emulation that relates NCC to GW for any choice of rate coefficients. In a similar fashion, we were able to show that there exists no triplet preserving emulation from NCC to DN and from CCR to MI.

Theorem 18. Let $(S, R)$ and $(\hat{S}, \hat{R})$ be unimodal influence networks and assume that $\mu: S \rightarrow \hat{S}$ is a triplet preserving emulation. Then, $\mu$ is also an emulation of the networks $\left(S, R^{\prime}\right)$ and $\left(\hat{S}, \hat{R}^{\prime}\right)$, where $R^{\prime}$ and $\hat{R}^{\prime}$ arise from $R$ and $\hat{R}$, respectively, by changing all rate coefficients to one.

Proof. Thanks to Theorem 17, we know that there exists a rate change $\iota: R \rightarrow R^{\prime \prime}$ such that $\mu$ is also an emulation of the networks $\left(S, R^{\prime \prime}\right)$ and $\left(\hat{S}, \hat{R}^{\prime}\right)$. We next show that all rate coefficients of $R^{\prime \prime}$ are equal to one, i.e. $R^{\prime \prime}=R^{\prime}$. To this end, we work on $\left(S, R^{\prime \prime}\right)$ and $\left(\hat{S}, \hat{R}^{\prime}\right)$. Let us fix some triplet $\left(x_{0}, x_{1}, x_{2}\right)$ in $(S, R)$ and assume first that $\left(\mu\left(x_{0}\right), \mu\left(x_{1}\right), \mu\left(x_{2}\right)\right)=\left(\hat{x}_{0}, \hat{x}_{1}, \hat{x}_{2}\right)$. Then, the reactions underlying node $x$ in $(S, R)$ are

$$
x_{0}+y_{i} \rightarrow{ }^{\alpha_{01}} y_{i}+x_{1}, \quad x_{1}+y_{i} \rightarrow{ }^{\alpha_{12}} y_{i}+x_{2}, \quad x_{2}+z_{j} \rightarrow{ }^{\alpha_{21}} z_{j}+x_{1}, \quad x_{1}+z_{j} \rightarrow{ }^{\alpha_{10}} z_{j}+x_{0},
$$

for some $i, j \in\{0,2\}$, whereas the reactions underlying node $\hat{x}$ in $\left(\hat{S}, \hat{R}^{\prime}\right)$ are

$$
\hat{x}_{0}+\hat{y}_{k} \rightarrow^{\hat{\alpha}_{01}} \hat{y}_{k}+\hat{x}_{1}, \quad \hat{x}_{1}+\hat{y}_{k} \rightarrow^{\hat{\alpha}_{12}} \hat{y}_{k}+\hat{x}_{2}, \quad \hat{x}_{2}+\hat{z}_{l} \rightarrow^{\hat{\alpha}_{21}} \hat{z}_{l}+\hat{x}_{1}, \quad \hat{x}_{1}+\hat{z}_{l} \rightarrow^{\hat{\alpha}_{10}} \hat{z}_{l}+\hat{x}_{0},
$$

for some $k, l \in\{0,2\}$. This yields

$$
\dot{v}_{x_{0}}=-\alpha_{01} v_{x_{0}} v_{y_{i}}+\alpha_{10} v_{x_{1}} v_{z_{j}} \quad \dot{\hat{v}}_{\hat{x}_{0}}=-\hat{\alpha}_{01} \hat{v}_{\hat{x}_{0}} \hat{v}_{\hat{y}_{k}}+\hat{\alpha}_{10} \hat{v}_{\hat{x}_{1}} \hat{v}_{\hat{z}_{l}}
$$

Let $v(0) \in \mathbb{R}^{S}$ be such that $v(0)=\hat{v}(0) \circ \mu$ where $\hat{v}_{\hat{a}_{1}}(0)=0$ and $\hat{v}_{\hat{a}_{0}}(0)=\hat{v}_{\hat{a}_{2}}(0)=1$ for all triplets $\left(\hat{a}_{0}, \hat{a}_{1}, \hat{a}_{2}\right)$ in $(\hat{S}, \hat{R})$. Since $\mu$ is an emulation, we know that $v_{x_{0}} \equiv \hat{v}_{\hat{x}_{0}}$, yielding $\dot{v}_{x_{0}} \equiv \dot{\hat{v}}_{\hat{x}_{0}}$. This and (5) imply

$$
-\alpha_{01} v_{x_{0}} v_{y_{i}}+\alpha_{10} v_{x_{1}} v_{z_{j}} \equiv-\hat{\alpha}_{01} \hat{v}_{\hat{x}_{0}} \hat{v}_{\hat{y}_{k}}+\hat{\alpha}_{10} \hat{v}_{\hat{x}_{1}} \hat{v}_{\hat{z}_{l}}
$$

Exploiting $v_{x_{0}} \equiv \hat{v}_{\hat{x}_{0}}$ and $v_{x_{2}} \equiv \hat{v}_{\hat{x}_{2}}$ yields $v_{x_{0}}\left(\hat{\alpha}_{01} \hat{v}_{\hat{y}_{k}}-\alpha_{01} v_{y_{i}}\right) \equiv \hat{v}_{\hat{x}_{1}}\left(\hat{\alpha}_{10} \hat{v}_{\hat{z}_{l}}-\alpha_{10} v_{z_{j}}\right)$. Since $v_{x_{0}}(0)=1$ and $\hat{v}_{\hat{x}_{1}}=0$, we get $\left(\hat{\alpha}_{01} \hat{v}_{\hat{y}_{k}}(0)-\alpha_{01} v_{y_{i}}(0)\right)=0$. This, the choice of $v$ and $\hat{v}$ and the fact that $k, i \in\{0,2\}$, in turn, imply that $\left(\hat{\alpha}_{01}-\alpha_{01}\right)=0$. Since $\hat{\alpha}_{01}=1$, this shows that $\alpha_{01}=1$.

Now, let $v(0) \in \mathbb{R}^{S}$ be such that $v(0)=\hat{v}(0) \circ \mu$ where $\hat{v}_{\hat{a}_{0}}(0)=\hat{v}_{\hat{a}_{1}}(0)=\hat{v}_{\hat{a}_{2}}(0)=1$ for all triplets $\left(\hat{a}_{0}, \hat{a}_{1}, \hat{a}_{2}\right)$ in $\left(\hat{S}, \hat{R}^{\prime}\right)$. Arguing as above, we infer that $v_{x_{0}}\left(\hat{\alpha}_{01} \hat{v}_{\hat{y}_{k}}-\alpha_{01} v_{y_{i}}\right) \equiv \hat{v}_{\hat{x}_{1}}\left(\hat{\alpha}_{10} \hat{v}_{\hat{z}_{l}}-\alpha_{10} v_{z_{j}}\right)$. By using the established relation $\alpha_{01}=\hat{\alpha}_{01}=1$ 
and evaluating the above equation for $t=0$, we infer that $0=\left(\hat{\alpha}_{10}-\alpha_{10}\right)$, thus showing $\hat{\alpha}_{10}=\alpha_{10}=1$. Similarly, it can be shown that $\dot{v}_{x_{2}}(0)=\dot{\hat{v}}_{\hat{x}_{2}}(0)$ implies that $\alpha_{21}=1$ and $\alpha_{12}=1$.

The case $\left(\mu\left(x_{0}\right), \mu\left(x_{1}\right), \mu\left(x_{2}\right)\right)=\left(\hat{x}_{2}, \hat{x}_{1}, \hat{x}_{0}\right)$ is shown in a similar fashion. Since the triplet $\left(x_{0}, x_{1}, x_{2}\right)$ was chosen arbitrarily, this shows the claim.

\subsection{Protein interaction networks}

In this section we apply CAGE to an implementation of the well-known repressilator system [27]. We consider a model distributed with BioNetGen [50], a rule-based language for modeling protein-interaction networks akin to Danos and Laneve's $\kappa$ calculus [4]. ${ }^{1}$ The model consists of three interacting transcriptional regulators: LacI, tet $R$, and $c I$. Each gene is modeled using three molecules:

$$
\operatorname{gTet} R(l, l), \operatorname{mTet} R(), p \operatorname{Tet} R(c), \quad g \operatorname{LacI}(c, c), \operatorname{mLacI}(), p \operatorname{LacI}(t), \quad g C I(t, t), \operatorname{mCI}(), p C I(l) .
$$

Each ' $g_{-}$' molecule produces the protein ' $p_{-}$' via the intermediate mRNA ' $m_{-}$'. In particular, $l, c$, and $t$ are the binding sites of the three molecules, used to bind to each other to form complex species. A term like $g$ Tet $R(l ! 1, l)$.pLacI(t!1) represents the complex species formed upon the binding of gTetR and pLacl (denoted by the '?' operator linking the two molecules, and '!1' which tags the sites through which the binding occurs). ${ }^{2}$

The genes are connected in a cycle of inhibitions: LacI inhibits the transcription of tet $R$, which in turn inhibits the expression of $c I$. Finally, $c I$ closes the cycle by inhibiting the expression of LacI. The CRN consists of 15 species and 30 reactions, with 5 species and 10 reactions per gene. The reactions (and species) for Tet $R$ are:

$$
\begin{aligned}
& g \operatorname{Tet} R(l, l)+p \operatorname{LacI}(t) \rightarrow^{2 \cdot r_{0}} g \operatorname{Tet} R(l ! 1, l) \cdot p \operatorname{LacI}(t ! 1) \\
& \operatorname{gTet} R(l ! 1, l) . p \operatorname{LacI}(t ! 1) \rightarrow^{r_{1}} \mathrm{gTet} R(l, l)+p \operatorname{LacI}(t) \\
& \operatorname{gTet} R(l ! 1, l) \cdot p \operatorname{LacI}(t ! 1)+p \operatorname{LacI}(t) \rightarrow^{r_{0}} g \operatorname{Tet} R(l ! 1, l ! 2) \cdot p \operatorname{LacI}(t ! 1) \cdot p \operatorname{LacI}(t ! 2) \\
& \operatorname{gTetR}(l ! 1, l ! 2) \cdot p \operatorname{LacI}(t ! 1) \cdot p \operatorname{LacI}(t ! 2) \rightarrow^{2 \cdot r_{2}} \mathrm{gTet} R(l ! 1, l) \cdot p \operatorname{LacI}(t ! 1)+p \operatorname{LacI}(t) \\
& \operatorname{gTet} R(l, l) \rightarrow^{r_{3}} \operatorname{gTet} R(l, l)+m \operatorname{Tet} R() \\
& \operatorname{gTetR}(l ! 1, l) \cdot p \operatorname{Lacl}(t ! 1) \rightarrow{ }^{r_{4}} \operatorname{gTet} R(l ! 1, l) \cdot p \operatorname{Lacl}(t ! 1)+\operatorname{mTet} R() \\
& \operatorname{gTet} R(l ! 1, l ! 2) \cdot p \operatorname{Lacl}(t ! 1) \cdot p \operatorname{Lacl}(t ! 2) \rightarrow^{r_{4}} \operatorname{gTet} R(l ! 1, l ! 2) \cdot p \operatorname{Lacl}(t ! 1) \cdot p \operatorname{Lacl}(t ! 2)+m \operatorname{Tet} R() \\
& m \operatorname{Tet} R() \rightarrow{ }^{r_{5}} \operatorname{mTet} R()+\operatorname{pTet} R(c) \\
& m \operatorname{Tet} R() \rightarrow{ }^{r_{6}} \emptyset \\
& p \operatorname{Tet} R(c) \rightarrow^{r_{7}} \emptyset
\end{aligned}
$$

Reactions (6)-(9) describe reversible bindings between tet $R$ and the product of LacI. This induces the discussed inhibition in the reactions (10)-(12), which produce $m$ TetR. Reaction (10), which refers to free gTetr molecules, has rate $r_{3}$. Instead, reactions (11)-(12), which refer to bound gTetr molecules, have rate $r_{4}$, where $r_{3}$ is three orders of magnitude larger than $r_{4}$. Finally, reaction (13) produces $p T e t r$, while reactions (14) and (15) consume $m T e t R$ and $p T e t R$, respectively. The reactions for LacI and $c I$ are not discussed because they have same structure and rates.

The coarsest BDE of the model consists of the following 6 blocks:

$$
\begin{aligned}
& \{\{g C I(t, t), g \operatorname{LacI}(c, c), g \operatorname{Tet} R(l, l)\}, \quad\{m C I(), m \operatorname{LacI}(), \operatorname{mTet} R()\}, \quad\{p \operatorname{Tet} R(c), p C I(l), p \operatorname{LacI}(t)\}, \\
& \{g C I(t ! 1, t) . p \operatorname{Tet} R(c ! 1), g \operatorname{LacI}(c ! 1, c) \cdot p C I(l ! 1), g \operatorname{Tet} R(l ! 1, l) \cdot p \operatorname{LacI}(t ! 1)\}, \\
& \{g C I(t ! 1, t ! 2) . p \operatorname{Tet} R(c ! 1) \cdot p \operatorname{Tet} R(c ! 2), g \operatorname{LacI}(c ! 1, c ! 2) \cdot p C I(l ! 1) . p C I(l ! 2), g \operatorname{Tet} R(l ! 1, l ! 2) . p \operatorname{LacI}(t ! 1) . p \operatorname{LacI}(t ! 2)\}\} .
\end{aligned}
$$

Hence, interestingly, BDE is able to identify symmetries in the genes dynamics due to the discussed cyclic inhibitions. Such BDE requires the three genes to be initialized with same concentration, while the intended oscillatory behavior is present when the genes have asymmetric initial concentrations. Hence, it is interesting to investigate if the model features further BDEs in presence of oscillatory behavior. By using CAGE we can answer this question negatively, as no further BDE is proven to exist.

\footnotetext{
1 The model is available at http://github.com/RuleWorld/bionetgen/blob/master/bng2/Validate/Repressilator.bngl.

2 Each $g_{-}$molecule has two copies of the same binding site. BioNetGen does not distinguish among species equal up to permutations of equivalent binding sites. Hence, e.g., $g \operatorname{Tet} R(l ! 1, l)$.pLacI $(t ! 1)$, and $g \operatorname{Tet} R(l, l ! 1)$.pLacI $(t ! 1)$ are equivalent, and only one of them will be generated.
} 


\section{Conclusion}

We have developed a framework for the comparison of CRNs based on the notion of emulation, i.e., a mapping from the species of a source CRN into those of a target CRN which establishes that related species have same concentration at any point in time. We characterized this semantic property in terms of structural conditions through flux morphisms. In addition to being useful in applications, this approach provides an explanation in terms of discrete, finitary, structures of behavior evolving as ordinary differential equations over continuous time and state spaces. Furthermore, we related emulation with backward differential equivalence. This is an equivalence relation over the species of a CRN which, similarly to emulation, establishes that equivalent species have same concentration at any point in time. Category theory gave use the language necessary for doing this, and all necessary constructions for this purpose have been provided. It would be interesting to investigate more general notions of equivalence, for instance such that are able to relate species which have same concentrations at any point in time, up to a given constant factor.

We discussed that the problem of finding emulations cannot be cast to the more traditional question of computing a largest equivalence (in this paper backward differential equivalence) that refines a given input partition. In fact, it is necessary to impose the constraint that every equivalence class contains exactly one species of the target CRN, and at least one of the source CRN. We addressed this problem by developing a new algorithm for computing emulations, based on a novel geometric interpretation of an equivalence relation for differential equations.

For quantitative notions of model comparison, it has long been argued that exact notions such as ours are too discriminant because of dependency on the choice of the numerical parameters, calling for approximate variants (e.g., [51-54]). We have started to tackle this issue by identifying a family of CRNs for which an emulation found for a given choice of parameters carries over to other values. In the special but biologically relevant case of (unimodal) influence networks, instead, we proved that the absence of an emulation for a specific choice of rates implies absence for any choice of rates. In the longer term, we believe that our contribution can be seen as a stepping stone in building approximate variants understood as appropriate perturbations on exact comparisons.

\section{Acknowledgements}

We thank the anonymous referees for helpful comments. This work was partially supported by the EU project QUANTICOL, 600708. L. Cardelli is partially funded by a Royal Society Research Professorship.

\section{References}

[1] A. Regev, E. Shapiro, Cellular abstractions: cells as computation, Nature 419 (6905) (2002) 343.

[2] G. Zavattaro, L. Cardelli, Termination problems in chemical kinetics, in: CONCUR, 2008, pp. 477-491.

[3] D. Doty, Timing in chemical reaction networks, in: SODA, 2014, pp. 772-784.

[4] V. Danos, C. Laneve, Formal molecular biology, Theoret. Comput. Sci. 325 (1) (2004) 69-110.

[5] J. Feret, V. Danos, J. Krivine, R. Harmer, W. Fontana, Internal coarse-graining of molecular systems, Proc. Natl. Acad. Sci. USA 106 (16) (2009) $6453-6458$.

[6] V. Danos, J. Feret, W. Fontana, R. Harmer, J. Krivine, Abstracting the differential semantics of rule-based models: exact and automated model reduction, in: LICS, 2010, pp. 362-381.

[7] F. Camporesi, J. Feret, H. Koeppl, T. Petrov, Combining model reductions, Electron. Notes Theor. Comput. Sci. 265 (2010) 73-96.

[8] J. Feret, T. Henzinger, H. Koeppl, T. Petrov, Lumpability abstractions of rule-based systems, Theoret. Comput. Sci. 431 (2012) $137-164$.

[9] L. Cardelli, M. Tribastone, M. Tschaikowski, A. Vandin, Forward and backward bisimulations for chemical reaction networks, in: CONCUR, 2015, pp. 226-239.

[10] L. Cardelli, Morphisms of reaction networks that couple structure to function, BMC Syst. Biol. 8 (1) (2014).

[11] L. Cardelli, A. Csikász-Nagy, N. Dalchau, M. Tribastone, M. Tschaikowski, Noise reduction in complex biological switches, Sci. Rep. 6 (2016) 20214.

[12] L. Cardelli, R.D. Hernansaiz-Ballesteros, N. Dalchau, A. Csikász-Nagy, Efficient switches in biology and computer science, PLoS Comput. Biol. 13 (1) (2017) $1-16$.

[13] D. Soloveichik, G. Seelig, E. Winfree, DNA as a universal substrate for chemical kinetics, Proc. Natl. Acad. Sci. USA 107 (12) (2010) $5393-5398$.

[14] L. Cardelli, M. Tribastone, M. Tschaikowski, A. Vandin, Symbolic computation of differential equivalences, in: POPL, 2016, pp. 137-150.

[15] J. Hillston, Fluid flow approximation of PEPA models, in: Proceedings of the Second International Conference on the Quantitative Evaluation of Systems, IEEE Computer Society Press, Torino, Italy, 2005, pp. 33-43.

[16] R.A. Hayden, J.T. Bradley, A fluid analysis framework for a Markovian process algebra, Theoret. Comput. Sci. 411 (22-24) (2010) $2260-2297$.

[17] M. Tribastone, S. Gilmore, J. Hillston, Scalable differential analysis of process algebra models, IEEE Trans. Softw. Eng. 38 (1) (2012) $205-219$.

[18] M. Tschaikowski, M. Tribastone, Exact fluid lumpability for Markovian process algebra, in: CONCUR, in: Lecture Notes in Comput. Sci., 2012, pp. 380-394.

[19] M. Tschaikowski, M. Tribastone, Tackling continuous state-space explosion in a Markovian process algebra, Theoret. Comput. Sci. 517 (2014) 1-33.

[20] M. Tschaikowski, M. Tribastone, Exact fluid lumpability in Markovian process algebra, Theoret. Comput. Sci. 538 (2014) $140-166$.

[21] M. Tschaikowski, M. Tribastone, A unified framework for differential aggregations in Markovian process algebra, J. Log. Algebraic Methods Program. 84 (2) (2015) 238-258.

[22] G. Iacobelli, M. Tribastone, A. Vandin, Differential bisimulation for a Markovian process algebra, in: MFCS, 2015, pp. 293-306.

[23] A. Vandin, M. Tribastone, Quantitative abstractions for collective adaptive systems, in: SFM 2016, Bertinoro Summer School, 2016, pp. 202-232.

[24] L. Cardelli, M. Tribastone, M. Tschaikowski, A. Vandin, Efficient syntax-driven lumping of differential equations, in: TACAS, 2016, pp. 93-111.

[25] L. Cardelli, M. Tribastone, M. Tschaikowski, A. Vandin, ERODE: a tool for the evaluation and reduction of ordinary differential equations, in: TACAS, 2017, pp. 310-328.

[26] J.J. Tyson, B. Novák, Functional motifs in biochemical reaction networks, Annu. Rev. Phys. Chem. 61 (2010) 219.

[27] M.B. Elowitz, S. Leibler, A synthetic oscillatory network of transcriptional regulators, Nature 403 (6767) (2000) 335-338.

[28] S. Gay, S. Soliman, F. Fages, A graphical method for reducing and relating models in systems biology, Bioinformatics 26 (18) (2010) i575-i581. 
[29] M. Lakin, A. Phillips, D. Stefanovic, Modular verification of DNA strand displacement networks via serializability analysis, in: DNA Computing and Molecular Programming, in: Lecture Notes in Comput. Sci., vol. 8141, 2013, pp. 133-146.

[30] S.W. Shin, C. Thachuk, E. Winfree, Verifying chemical reaction network implementations: a pathway decomposition approach, in: VEMDP, Vienna Summer of Logic, 2014, pp. 67-89.

[31] M. Lakin, D. Stefanovic, A. Phillips, Modular verification of chemical reaction network encodings via serializability analysis, Theoret. Comput. Sci. 632 (2016) 21-42.

[32] M. Boreale, Weighted bisimulation in linear algebraic form, in: CONCUR, 2009, pp. 163-177.

[33] M. Boreale, Algebra, coalgebra, and minimization in polynomial differential equations, in: FOSSACS, 2017, pp. 71-87.

[34] G. Li, H. Rabitz, A general analysis of exact lumping in chemical kinetics, Chem. Eng. Sci. 44 (6) (1989) 1413-1430.

[35] N.M. Borisov, N.I. Markevich, J.B. Hoek, B.N. Kholodenko, Signaling through receptors and scaffolds: independent interactions reduce combinatorial complexity, Biophys. J. 89 (2) (2005) 951-966.

[36] H. Conzelmann, J. Saez-Rodriguez, T. Sauter, B. Kholodenko, E. Gilles, A domain-oriented approach to the reduction of combinatorial complexity in signal transduction networks, BMC Bioinform. 7 (1) (2006) 34

[37] H. Conzelmann, D. Fey, E. Gilles, Exact model reduction of combinatorial reaction networks, BMC Syst. Biol. 2 (1) (2008) 78.

[38] A. van der Schaft, Equivalence of dynamical systems by bisimulation, IEEE Trans. Automat. Control 49 (12) (2004) $2160-2172$.

[39] L. Cardelli, M. Tribastone, M. Tschaikowski, A. Vandin, Comparing chemical reaction networks: a categorical and algorithmic perspective, in: LICS, 2016, pp. 485-494.

[40] S. Tognazzi, M. Tribastone, M. Tschaikowski, A. Vandin, EGAC: a genetic algorithm to compare chemical reaction networks, in: Proceedings of the Genetic and Evolutionary Computation Conference, GECCO 2017, Berlin, Germany, July 15-19, 2017, 2017, pp. 833-840.

[41] E.O. Voit, Biochemical systems theory: a review, ISRN Biomath. 2013 (2013), https://doi.org/10.1155/2013/897658, 53 pp.

[42] M. Hirsch, S. Smale, Differential Equations, Dynamical Systems, and Linear Algebra, Pure Appl. Math., Acad. Press, 1974.

[43] L. Perko, Differential Equations and Dynamical Systems, Springer, New York, 1991.

[44] M. Domijan, What are...some graphs of chemical reaction networks?, available at http://www.math.uzh.ch/?file\&key1=9116.

[45] L. Cardelli, A. Csikász-Nagy, The cell cycle switch computes approximate majority, Sci. Rep. 2 (2012) 656.

[46] A. Verdugo, P.K. Vinod, J.J. Tyson, B. Novak, Molecular mechanisms creating bistable switches at cell cycle transitions, Open Biol. 3 (3) (2013) 120179.

[47] T.S. Gardner, C.R. Cantor, J.J. Collins, Construction of a genetic toggle switch in Escherichia coli, Nature 403 (6767) (2000) 339-342.

[48] B. Novak, J. Tyson, Numerical analysis of a comprehensive model of m-phase control in Xenopus oocyte extracts and intact embryos, J. Cell Sci. 106 (4) (1993) 1153-1168.

[49] R. Ghosh, C. Tomlin, Lateral inhibition through Delta-Notch signaling: a piecewise affine hybrid model, in: Hybrid Systems: Computation and Control, Proceedings of the 4th International Workshop, HSCC 2001, Rome, Italy, March 28-30, 2001, 2001, pp. 232-246.

[50] M.L. Blinov, J.R. Faeder, B. Goldstein, W.S. Hlavacek, BioNetGen: software for rule-based modeling of signal transduction based on the interactions of molecular domains, Bioinformatics 20 (17) (2004) 3289-3291.

[51] J. Desharnais, V. Gupta, R. Jagadeesan, P. Panangaden, Metrics for labeled Markov systems, in: CONCUR, 1999, pp. 258-273.

[52] F. van Breugel, J. Worrell, Approximating and computing behavioural distances in probabilistic transition systems, Theoret. Comput. Sci. 360 (1-3) (2006) 373-385.

[53] J. Desharnais, V. Gupta, R. Jagadeesan, P. Panangaden, Metrics for labelled Markov processes, Theoret. Comput. Sci. 318 (3) (2004) $323-354$.

[54] K.G. Larsen, R. Mardare, P. Panangaden, Taking it to the limit: approximate reasoning for Markov processes, in: MFCS, 2012, pp. 681-692. 